Supporting Information for

\title{
On the Origin of the Photostability of DNA and RNA Monomers: Excited State Relaxation Mechanism of the Pyrimidine Chromophore
}

\author{
Enrique M. Arpa ${ }^{1}$, Matthew M. Brister ${ }^{2,3}$, Sean J. Hoehn ${ }^{2}$, Carlos E. Crespo-Hernández ${ }^{2}$, Inés \\ Corral $^{1,4}$ \\ ${ }^{1}$ Dpto. Química. Facultad de Ciencias. Universidad Autónoma de Madrid. C/ Francisco Tomás y Valiente 7, \\ 28049 Madrid, España. \\ 2 Department of Chemistry, Case Western Reserve University, Cleveland, Ohio 44106, United \\ States. \\ ${ }^{3}$ Current Address: Lawrence Berkeley National Laboratory, Atomic Molecular and Optical Sciences, Chemical \\ Science Division, Berkeley, CA 94720, United States. \\ ${ }^{4}$ Institute of Advanced Research in Chemical Sciences (IAdChem). Universidad Autónoma de Madrid. C/ \\ Francisco Tomás y Valiente 7, 28049 Madrid, España.
}

\section{Table of contents}

1. Computational Details S1

2. Steady-state Properties of Pyrimidine S2

3. Relevant geometries

4. Generation of the Individual Transient Absorption Spectrum for Each Minimum

5. Modeling of Evolution Associated Difference Spectra from Calculated Data

6. Experimental Methodology

7. References 


\section{Computational Details}

Optimization of the ground state geometry was performed at the MP2/cc-pVTZ level of theory using Gaussian 09 package ${ }^{1}$.

For the modeling of the gas phase absorption spectrum, vertical excitation energies at the Franck-Condon region were calculated at the MS-CASPT2 (multi-state second-order perturbation theory ${ }^{2}$ ) level of theory considering SA-CASSCF wavefunctions (state-average complete active space self-consistent field ${ }^{3}$ ), over 20 singlet and 20 triplet states, as a reference, in combination with the ANO-L-VTZ basis set ${ }^{4}$. The employed active space $(10,8)$ included the complete $\pi$ system of the pyrimidine and the two $N$ lone-pairs (see Figure S1).

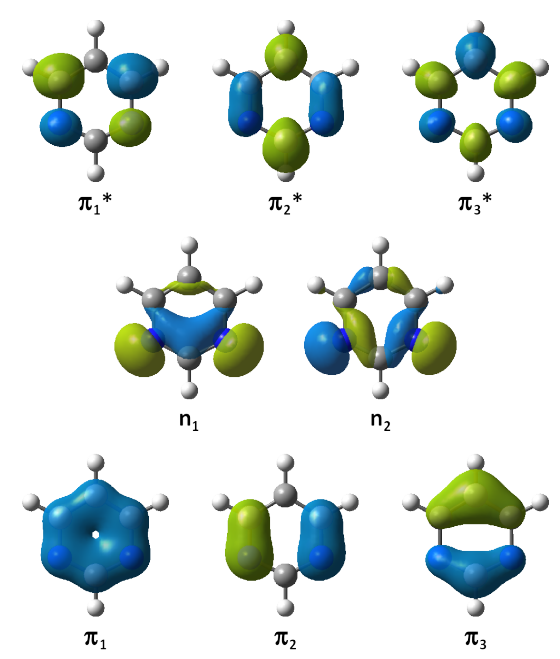

Scheme S1 CASSCF orbitals at the $\mathrm{S}_{0}$ minimum included in the active space used throughout this work.

Minimum Energy Path calculations were carried out to locate accessible minima or surface crossings at the SA-CASSCF(10,8)/ANO-L-VTZ level of theory (see Section 3 for further information of the number of roots employed), the same protocol was used for optimization of the singlet and ${ }^{3} \pi \pi^{*}$ minima.

The location of $S_{1} / S_{0}$ minimum energy crossing points was carried out at the XMS-CASPT2/cc-pVTZ/cc-pVTZ (extended multi-state CASPT $2^{5}$ with cc-pVTZ basis set and cc-pVTZ auxiliary basis set for density-fitting) level of theory using $B A G E L^{6}$. The same method was used to locate ${ }^{3} n \pi^{*}$ minima. On the other hand, the $S_{1} / T_{2}$ crossing point was located at the SA-CASSCF(10,8)/6-31G* level using MOLPRO 2015.1 ${ }^{7}$. Spin-orbit coupling at the $S_{1} / T_{2}$ minimum energy crossing point was calculated at the MS-CASPT2/ANO-RCC-VDZP level of theory to qualitatively estimate the probability of intersystem crossing.

Final energies of all stationary points and interstate crossings (Figure 4) were computed at the MS-CASPT2/ANO-L-VTZ over the SA-CASSCF/ANO-L-VTZ wavefunctions (4 singlet or 5 triplet states).

Transient Absorption Spectra were computed at the MS-CASPT2/ANO-L-VTZ level of theory at the corresponding minima, twenty states (singlets or triplets) were considered. Here the IPEA shift was set to zero. The weight of intruder states in multistate CASPT2 calculations was set manually to zero.

Although otherwise indicated, the default IPEA shift and a 0.25 au level shift in the Oth order Hamiltonian were used in all the calculations, performed with MOLCAS 7.8 software $^{8}$. 


\section{Steady-state Properties of Pyrimidine}

\subsection{Solvatochromism of the Ground State Absorption Spectrum}

The experimental absorption spectra of pyrimidine (see Figure 1) showed that the lowest-energy peak, absorbing at $293 \mathrm{~nm}$ in cyclohexane, undergoes a blue shift ( $288 \mathrm{~nm}$ in MeCN, $270 \mathrm{~nm}$ in water) as the polarity of the solvent increases, whereas the highest-energy peak $(243 \mathrm{~nm})$ remains unaltered. To rationalize these solvatochromic effects the vertical absorption spectrum was computed at the TD-CAM-B3LYP/aug-cc-pVTZ/PCM level of theory ${ }^{9-10}$ in 7 different solvents (water, dimethylsulfoxide, acetonitrile, acetone, dichloromethane, chloroform, o-xylene and cyclohexane) over the ground state geometry (MP2/cc-pVTZ in gas phase). In all cases, the character of the excitations is in agreement with the MS-CASPT2 results in the gas-phase: the lowest energy band corresponds to a $S_{0} \rightarrow S_{1}\left(n_{2} \pi_{1}^{*}\right.$, see Scheme S1) transition, whereas the second one corresponds to a $S_{0} \rightarrow S_{3}\left(\pi_{3} \pi_{1}{ }^{*}\right)$ transition. Interestingly, the wavelength of the $S_{0} \rightarrow S_{1}$ transition linearly correlates with the inverse of epsilon. Our calculations are also able to reproduce the bathochromic effect over the lowest enegy peak upon the decrease of the solvent polarity (See Figure S1, right). Therefore the higher the polarity of the solvent, the higher the energy of the transition will be. On the other hand, the energy of the $S_{0} \rightarrow S_{3}$ transition does not change substantially with any of the solvents considered (see Figure S1, left).

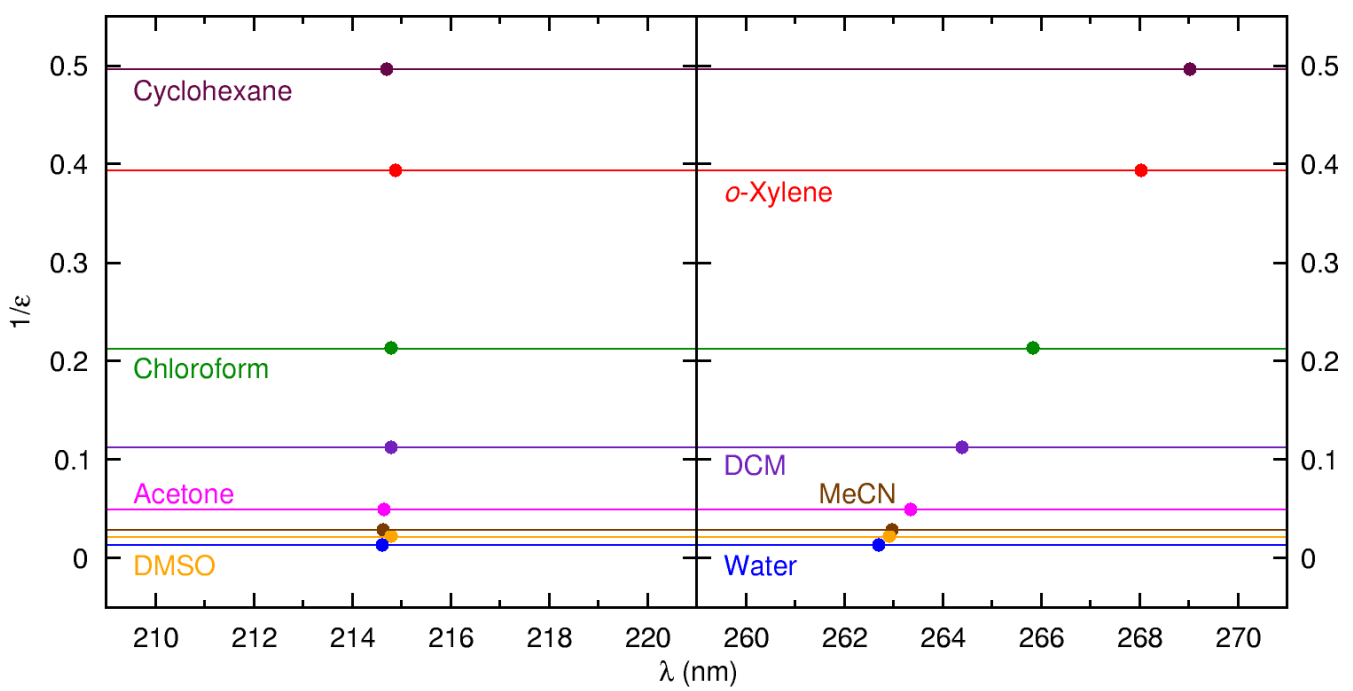

Figure S1 Correlation between wavelengths of the $S_{0} \rightarrow S_{1}$ (right) and $S_{0} \rightarrow S_{3}$ (left) transitions and solvent polarity.

Consistently, these solvatochromic effects correlate with the variation of the dipole moment along the excitation. In fact, the $S_{1}$ state was computed to be less polar than the ground state, whilst the $S_{3}$ shows a similar dipole moment compared to the $S_{0}$ (see Table $S 2$ ).

\begin{tabular}{|c|c|c|c|}
\hline State & $\mathrm{S}_{0}$ & \\
\hline$\mu(\mathrm{D})$ & 2.43 & \\
\hline & &
\end{tabular}

Table S1 Dipole moments for $\mathrm{S}_{0}, \mathrm{~S}_{1}$ and $\mathrm{S}_{3}$ states in the Franck-Condon region. 


\subsection{Excitation and emission spectra and calculation of 0-0 energy}

The emission and excitation spectra were used to determine the 0-0 energy (Figure S2) and Table S1. The 0-0 energy was also estimated from the normalized emission and absorption spectra. From the excitation spectra it was corroborated that the fluorescence emission occurs from the lowest energy absorption band of pyrimidine.

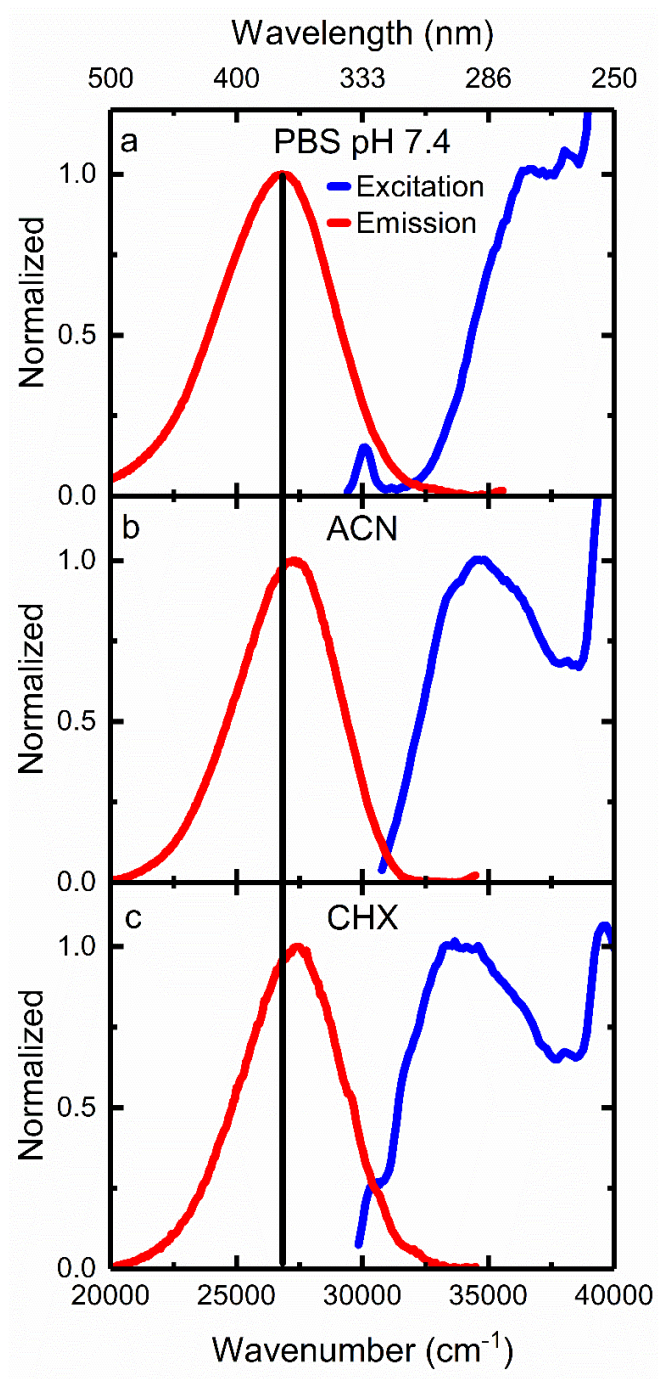

Figure S2 Excitation and emission spectra of pyrimidine in PBS (upper), MeCN (middle) and cyclohexane (CHX, lower)

\begin{tabular}{|c|c|c|c|c|c|}
\hline Solvent & $\lambda_{\text {Abs }}( \pm 1 \mathrm{~nm})$ & $\varepsilon\left( \pm 200 \mathrm{M}^{-1} \mathrm{~cm}^{-1}\right)$ & $\lambda_{\mathrm{Fl}}( \pm 5 \mathrm{~nm})$ & $\mathrm{Em} / \mathrm{Ex}_{\mathrm{o}-0}(\mathrm{eV})$ & Em/Abs $E_{0-0}(e V)$ \\
\hline PBS & $\begin{array}{l}243 \\
270\end{array}$ & $\begin{array}{c}4762 \\
690\end{array}$ & 374 & 3.96 & 4.00 \\
\hline $\mathrm{MeCN}$ & $\begin{array}{l}243 \\
288\end{array}$ & $\begin{array}{c}3978 \\
695\end{array}$ & 367 & 3.84 & 3.86 \\
\hline $\mathrm{CHX}$ & $\begin{array}{l}244 \\
293\end{array}$ & --- & 365 & 3.78 & 3.86 \\
\hline
\end{tabular}

Table S2 Summary of steady-state properties of pyrimidine. Abs: Absorption, Em: Emission, Ex: Excitation, Fl: Fluorescence 


\section{Relevant Geometries}

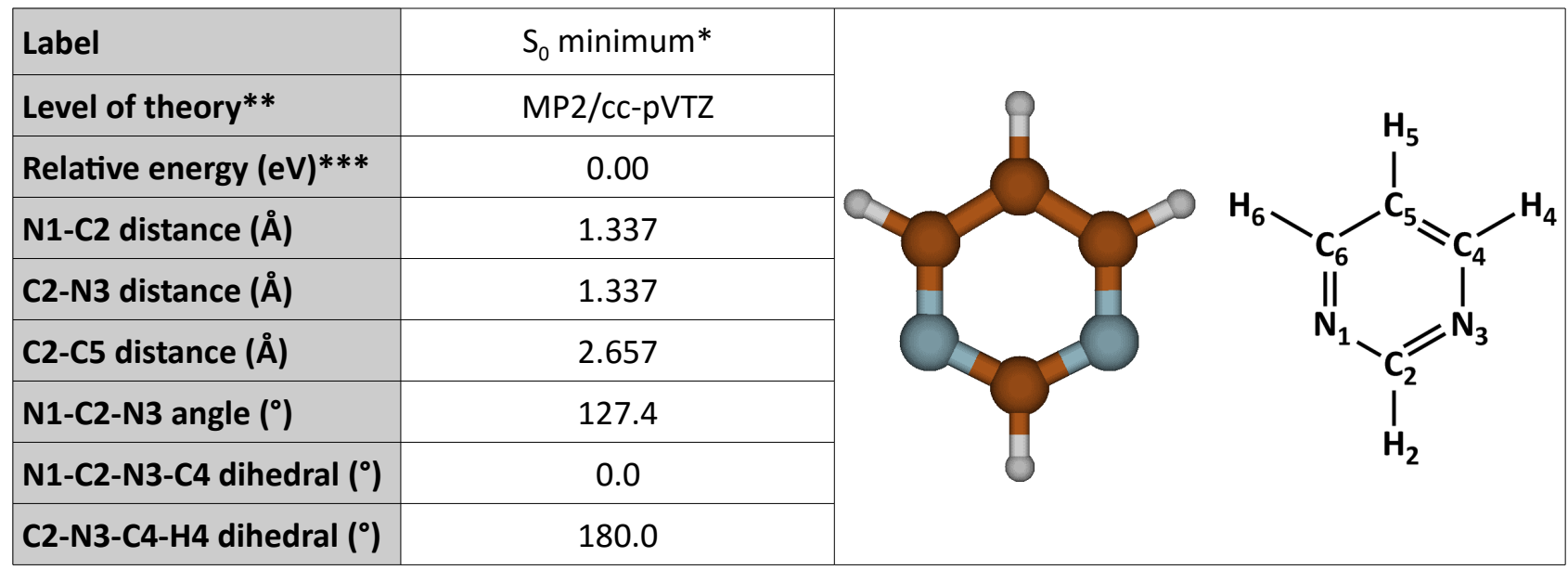

* Atom labelling scheme used throughout all the structures

** For geometry optimization

*** Energies were calculated at the MS-CASPT2//SA-CASSCF(10,8)/ANO-L level of theory, with 4 states for singlets and 5 states for triplets

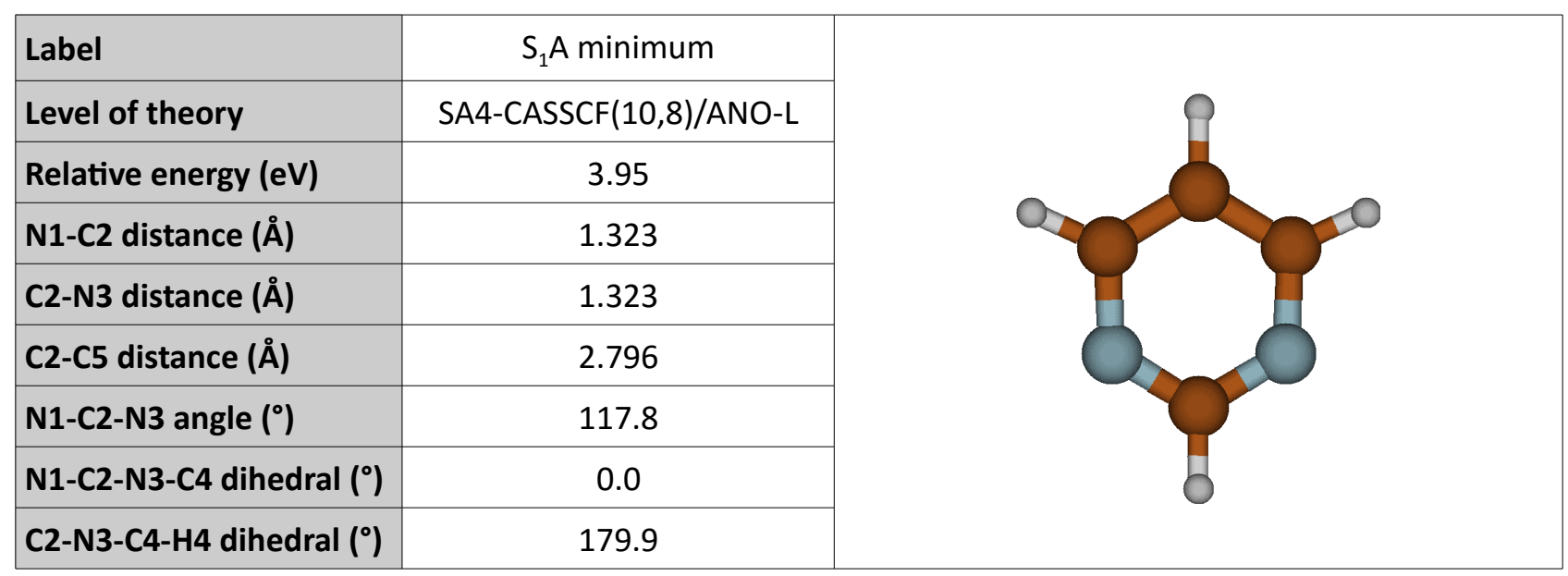

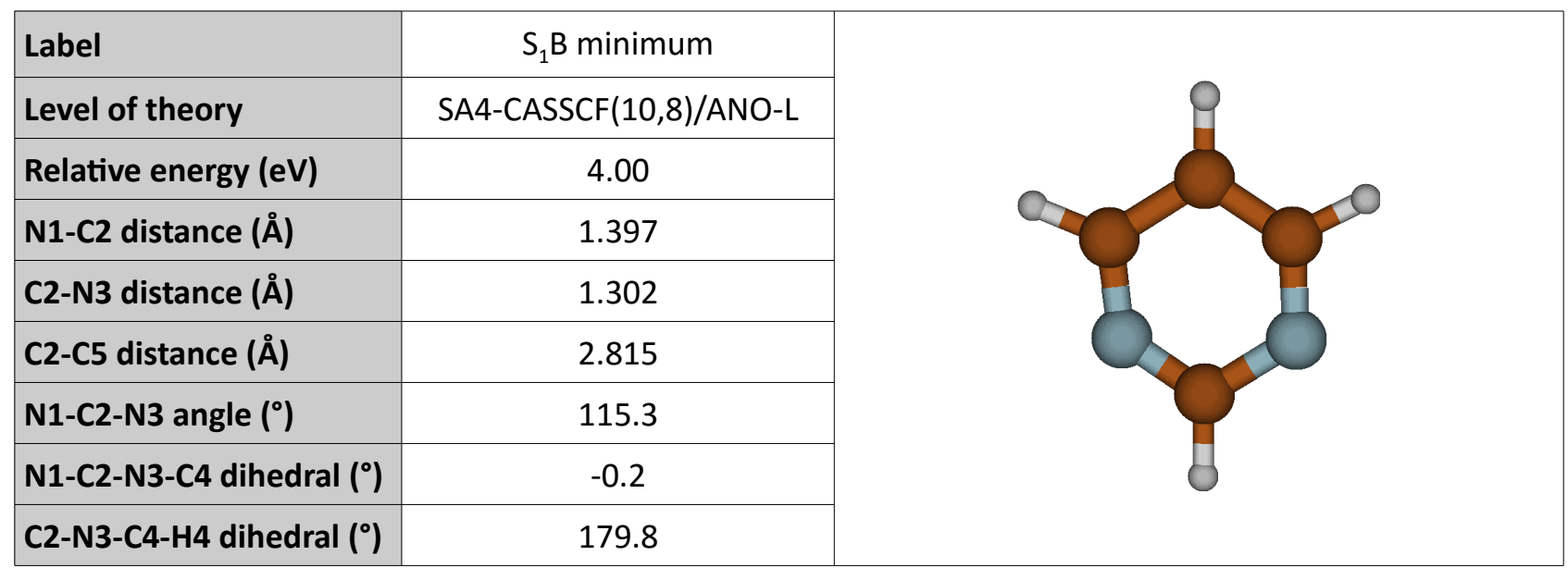




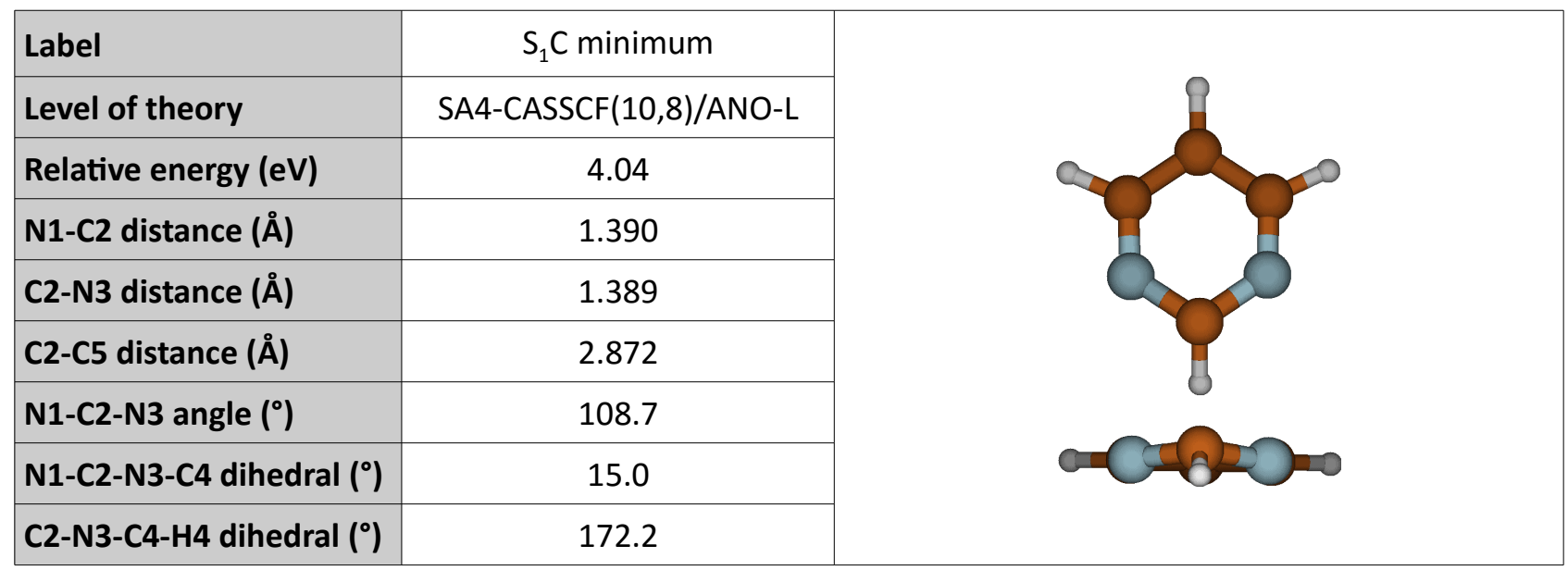

\begin{tabular}{|l|c|}
\hline Label & $\mathrm{T}_{1} \mathrm{~A}$ minimum \\
\hline Level of theory & SA5-CASSCF(10,8)/ANO-L \\
\hline Relative energy (eV) & 3.96 \\
\hline N1-C2 distance $(\AA \AA)$ & 1.406 \\
\hline C2-N3 distance $(\stackrel{\AA}{\mathbf{A}})$ & 1.277 \\
\hline C2-C5 distance $(\AA)$ & 2.711 \\
\hline N1-C2-N3 angle $\left(^{\circ}\right)$ & 127.1 \\
\hline N1-C2-N3-C4 dihedral $\left(^{\circ}\right)$ & 5.8 \\
\hline C2-N3-C4-H4 dihedral $\left(^{\circ}\right)$ & 121.9 \\
\hline
\end{tabular}

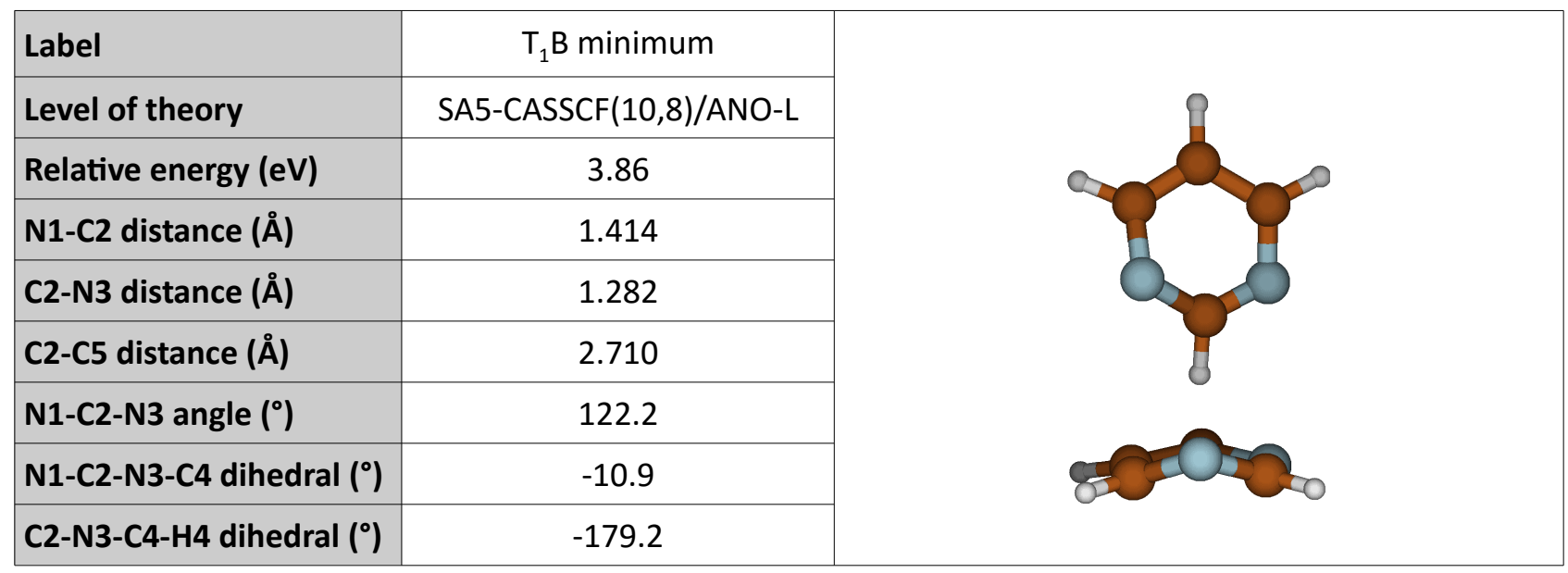




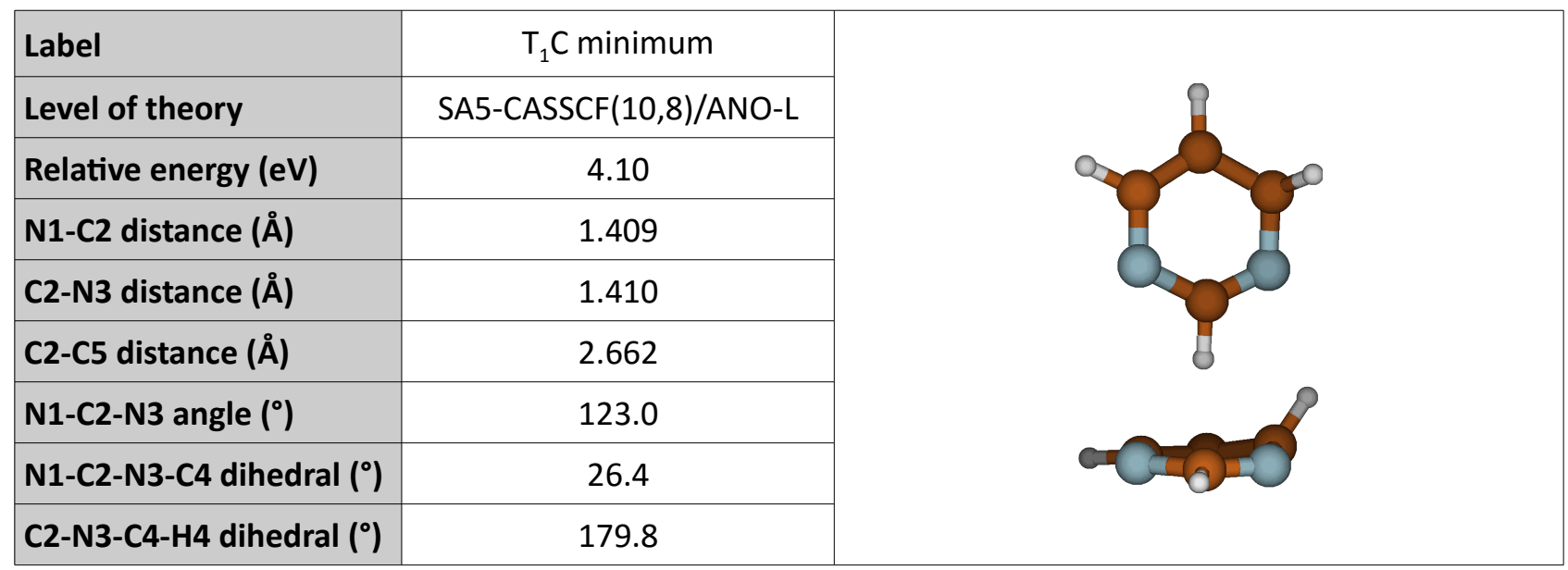

\begin{tabular}{|l|c|}
\hline Label & $\mathrm{T}_{1} \mathrm{D}$ minimum \\
\hline Level of theory & SA5-CASSCF(10,8)/ANO-L \\
\hline Relative energy (eV) & 3.96 \\
\hline N1-C2 distance $(\AA \AA)$ & 1.432 \\
\hline C2-N3 distance $(\stackrel{\AA}{\mathbf{A}})$ & 1.432 \\
\hline C2-C5 distance $(\AA)$ & 2.655 \\
\hline N1-C2-N3 angle $\left(^{\circ}\right)$ & 121.0 \\
\hline N1-C2-N3-C4 dihedral $\left(^{\circ}\right)$ & 32.8 \\
\hline C2-N3-C4-H4 dihedral $\left(^{\circ}\right)$ & 177.0 \\
\hline
\end{tabular}

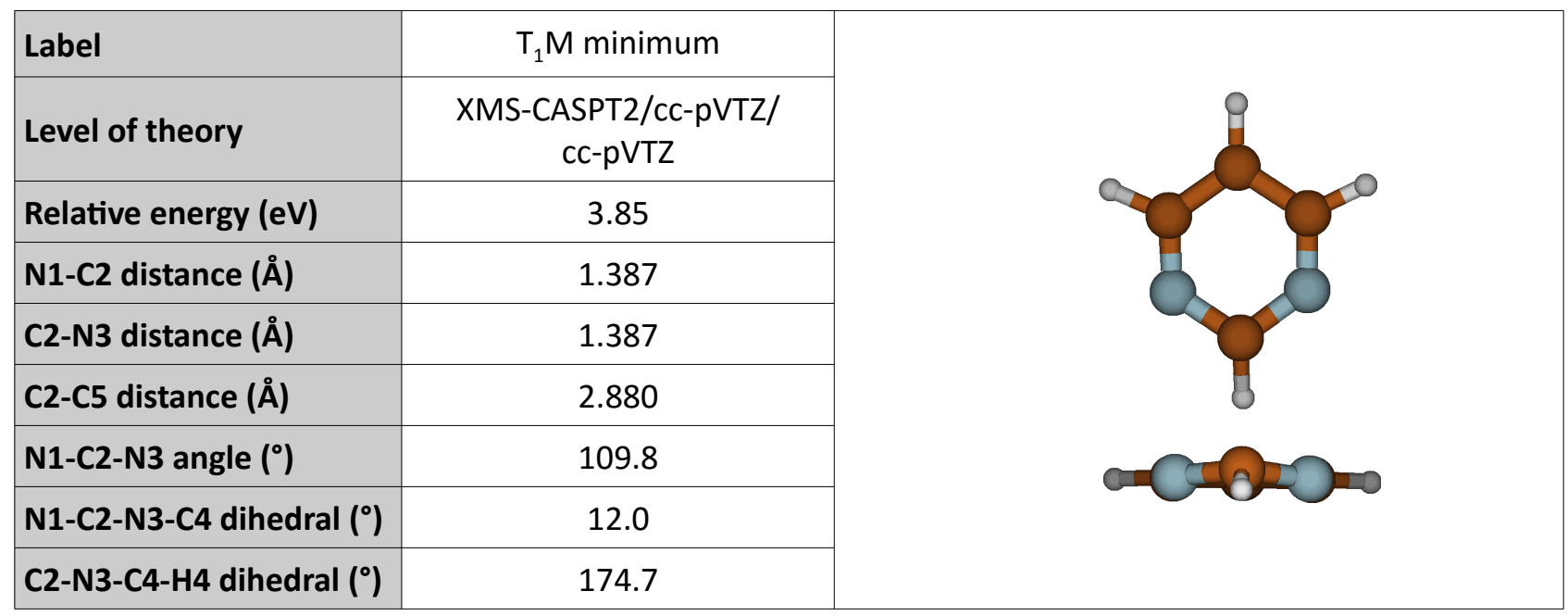




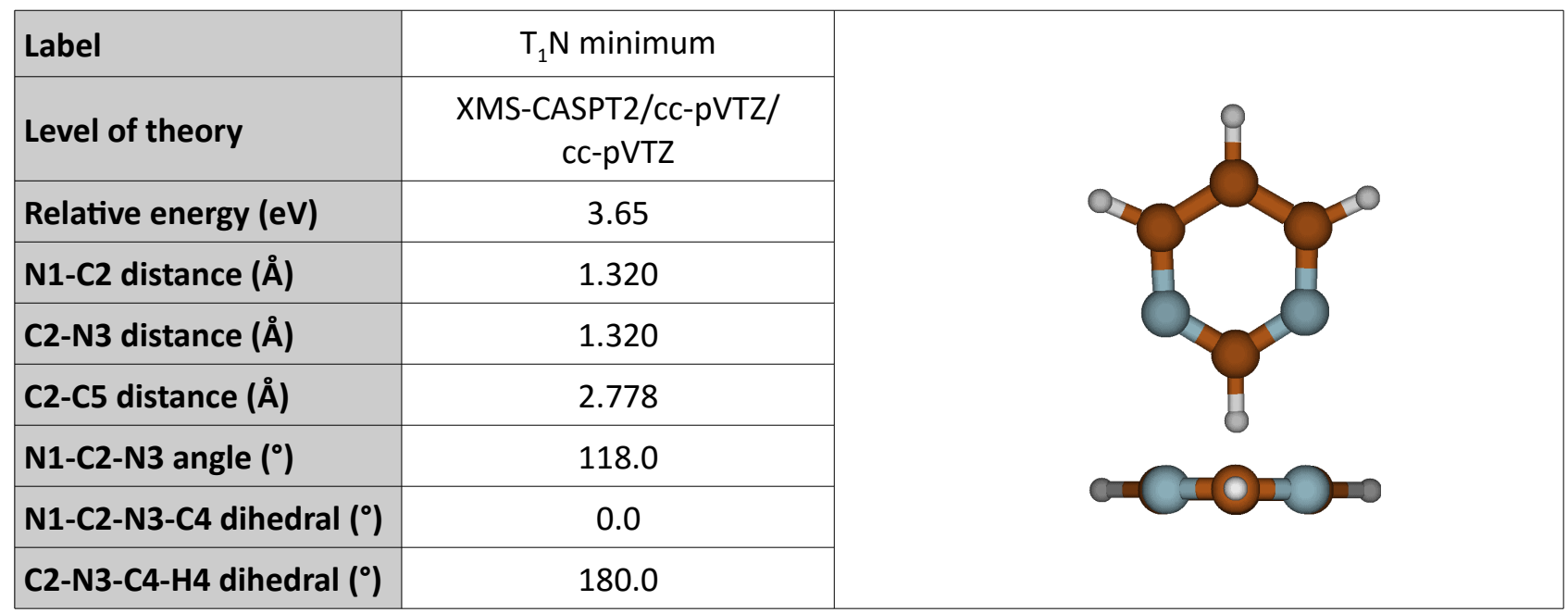

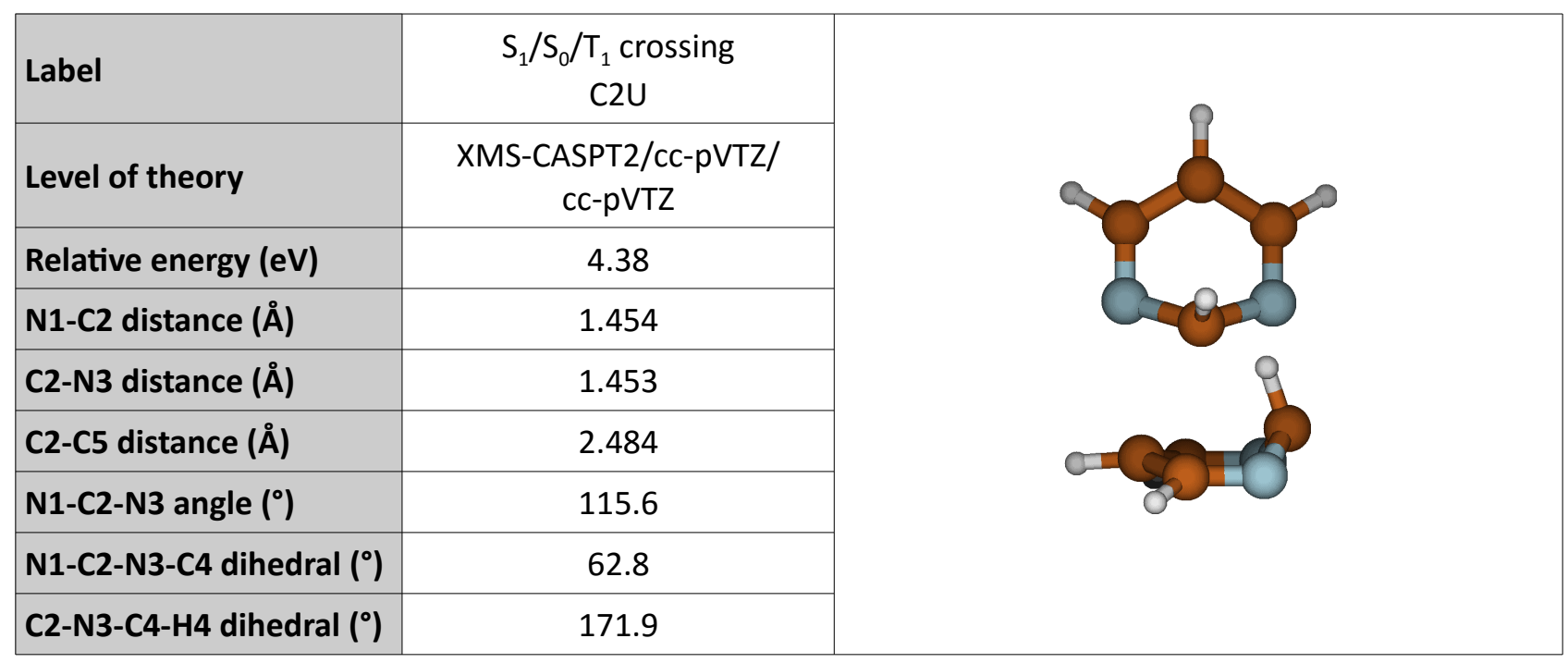

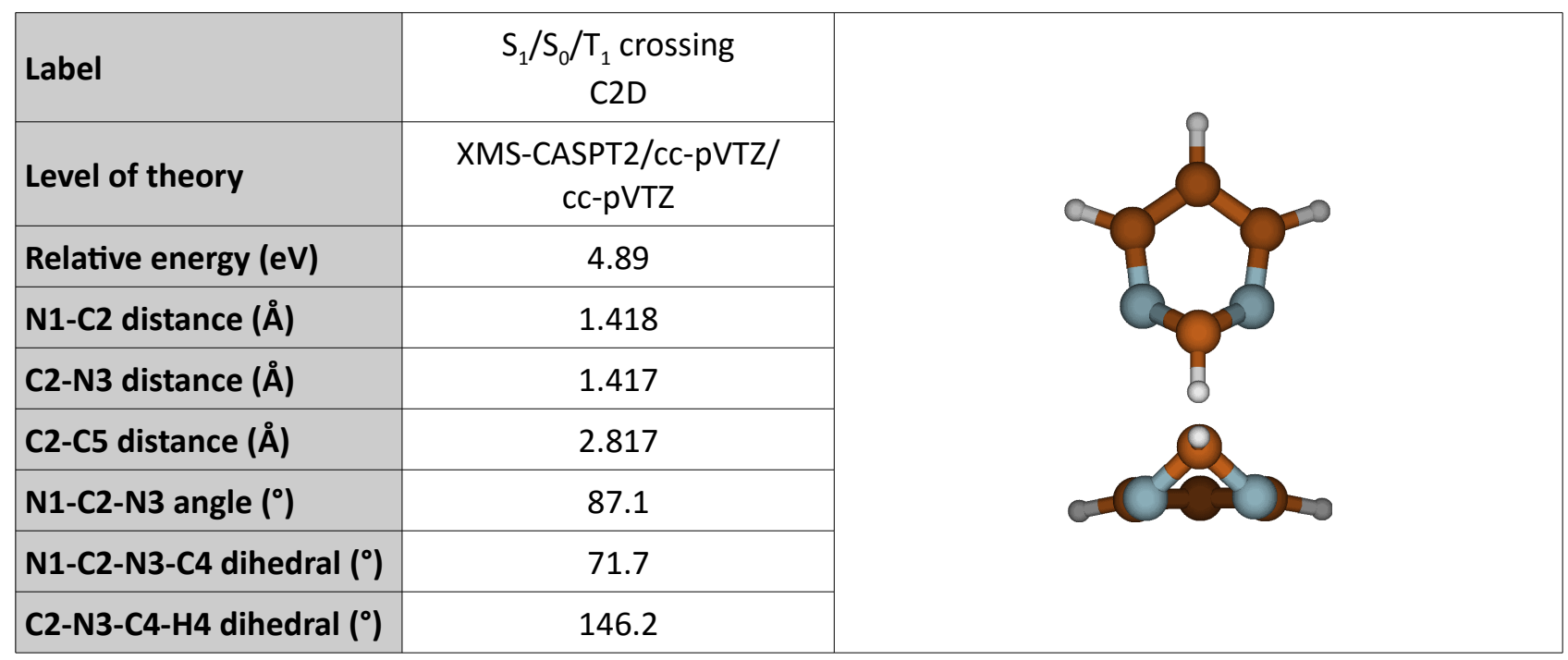




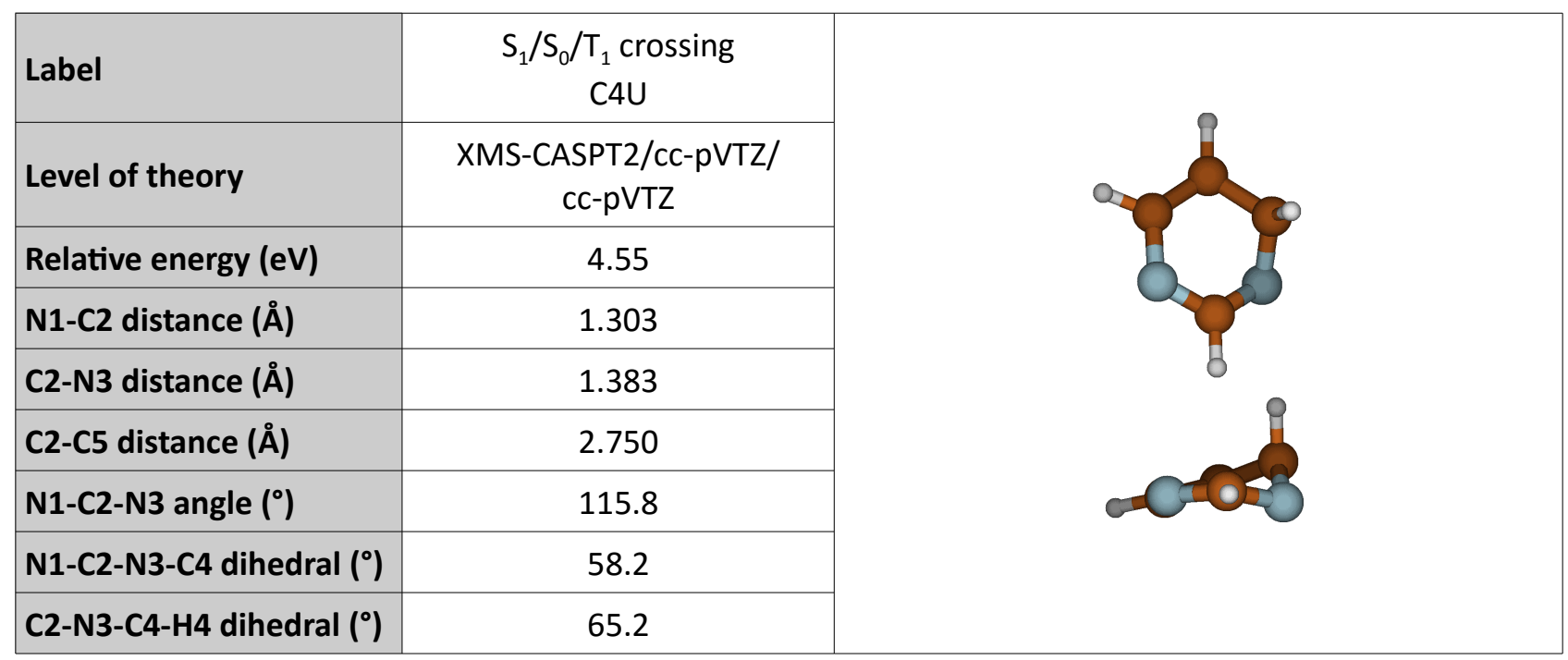

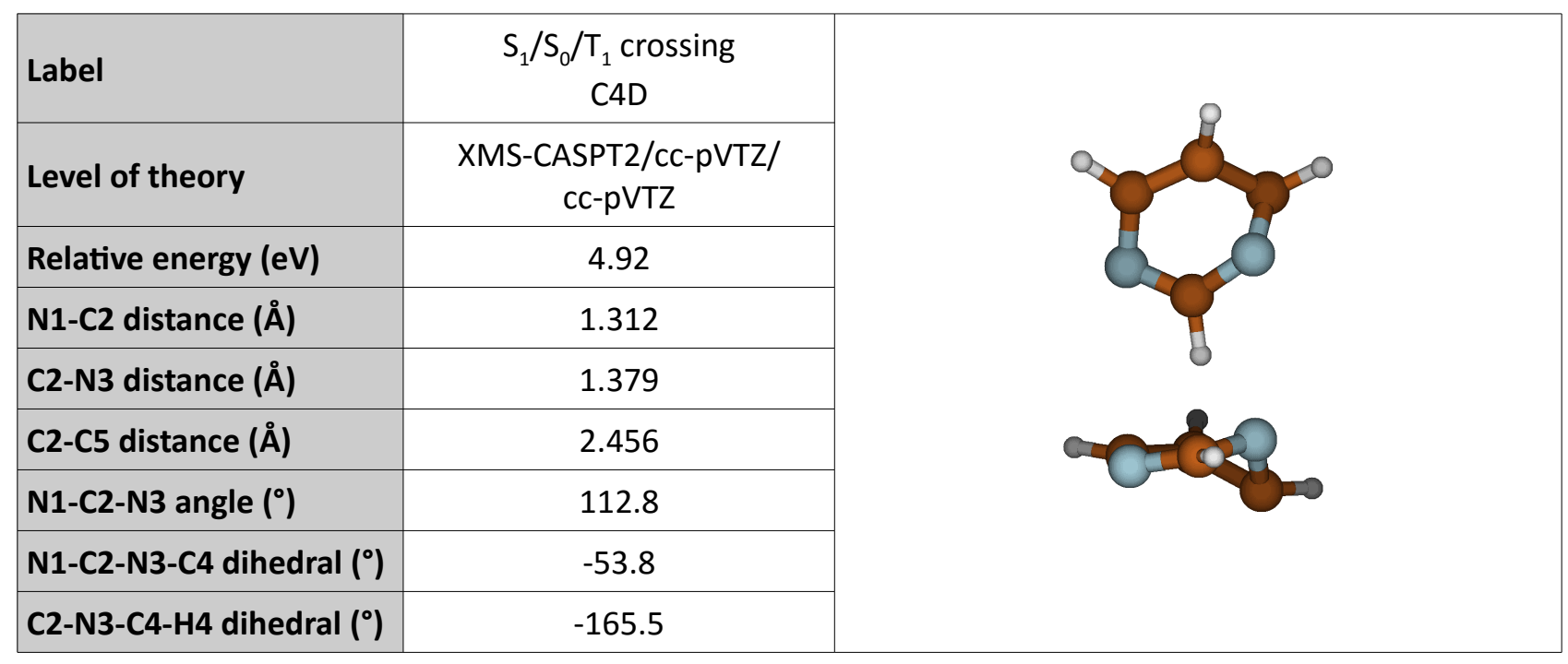

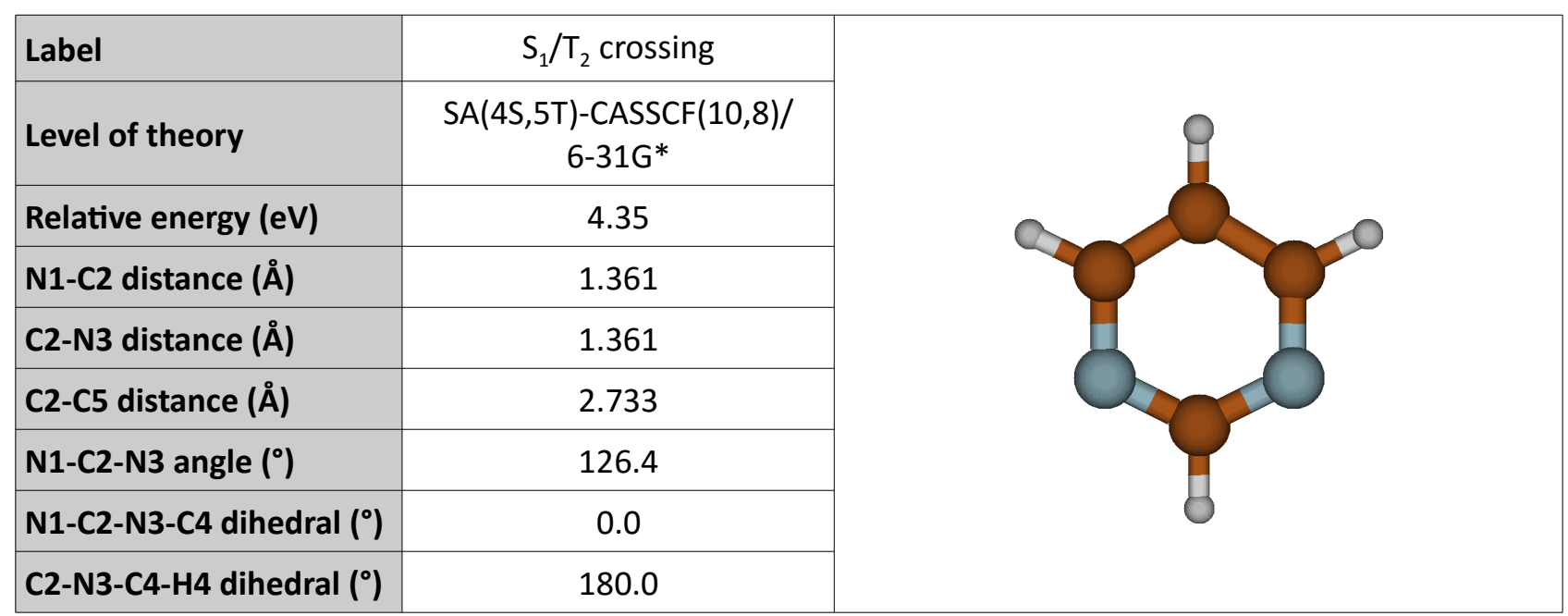




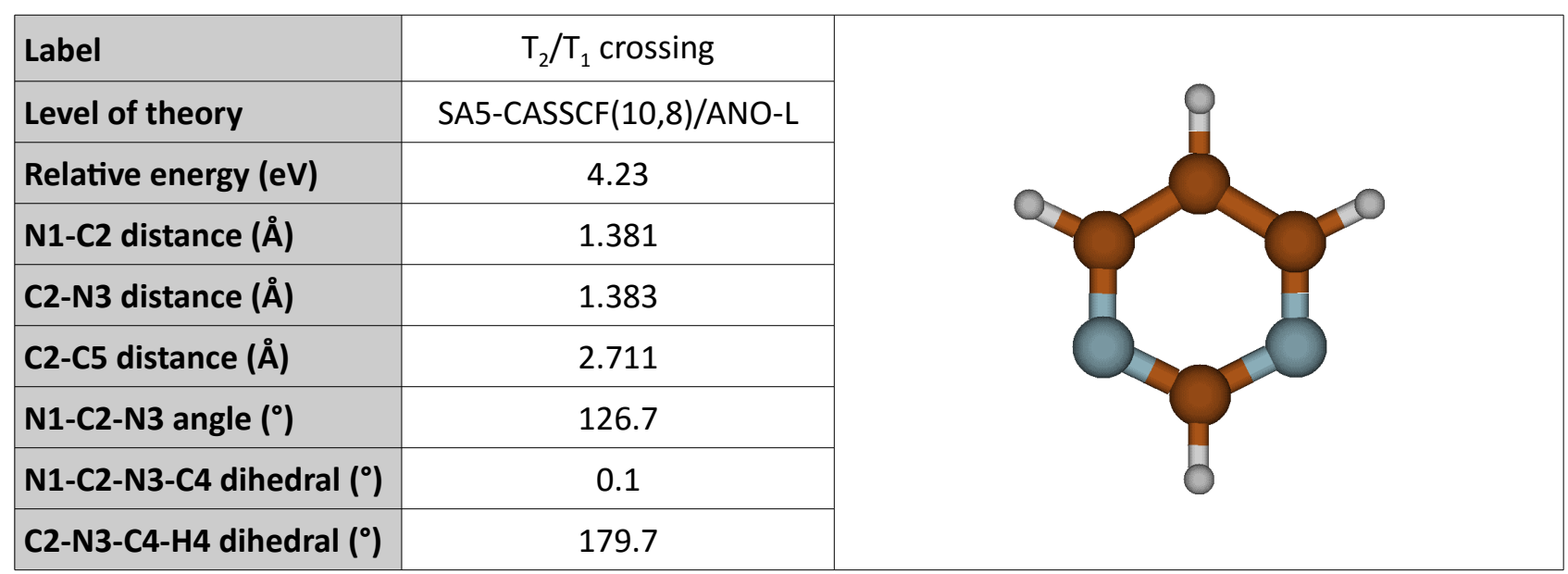




\section{Generation of the Individual Transient Absorption Spectrum for each minimum}

Simulated TAS were obtained from the vertical excitation energies and their corresponding oscillator strengths at each excited minimum using the line broadening method, that is assigning to each excitation a Gaussian function whose center is located on the excitation energy and whose maximum height is equal to the oscillator strength. The final spectrum is built as the sum of all Gaussian functions (see Figures S3-S11). The half-width of all Gaussian functions was set to $50 \mathrm{~nm}$. This procedure was done with the Gabedit 2.4.8 program.

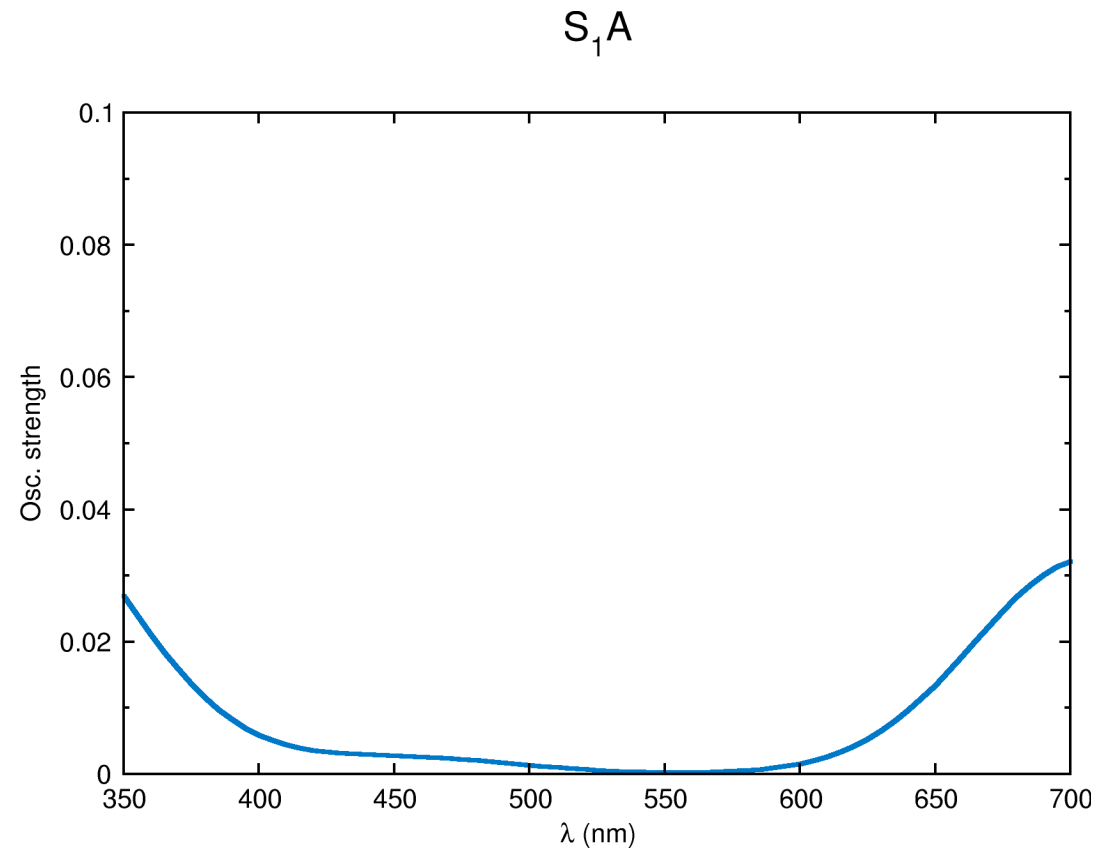

Figure S3 Transient Absorption Spectrum for $S_{1} A$ minimum

\section{$S_{1} B$}

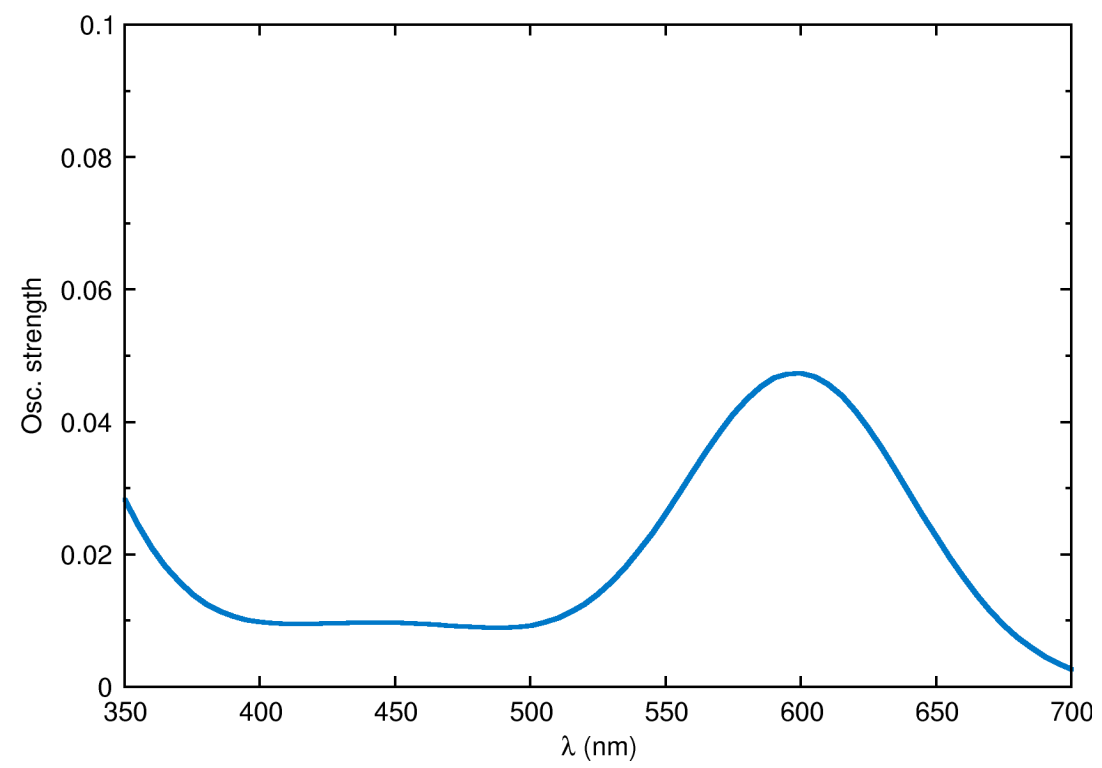

Figure S4 Transient Absorption Spectrum for $\mathrm{S}_{1} \mathrm{~B}$ minimum 
$\mathrm{S}_{1} \mathrm{C}$

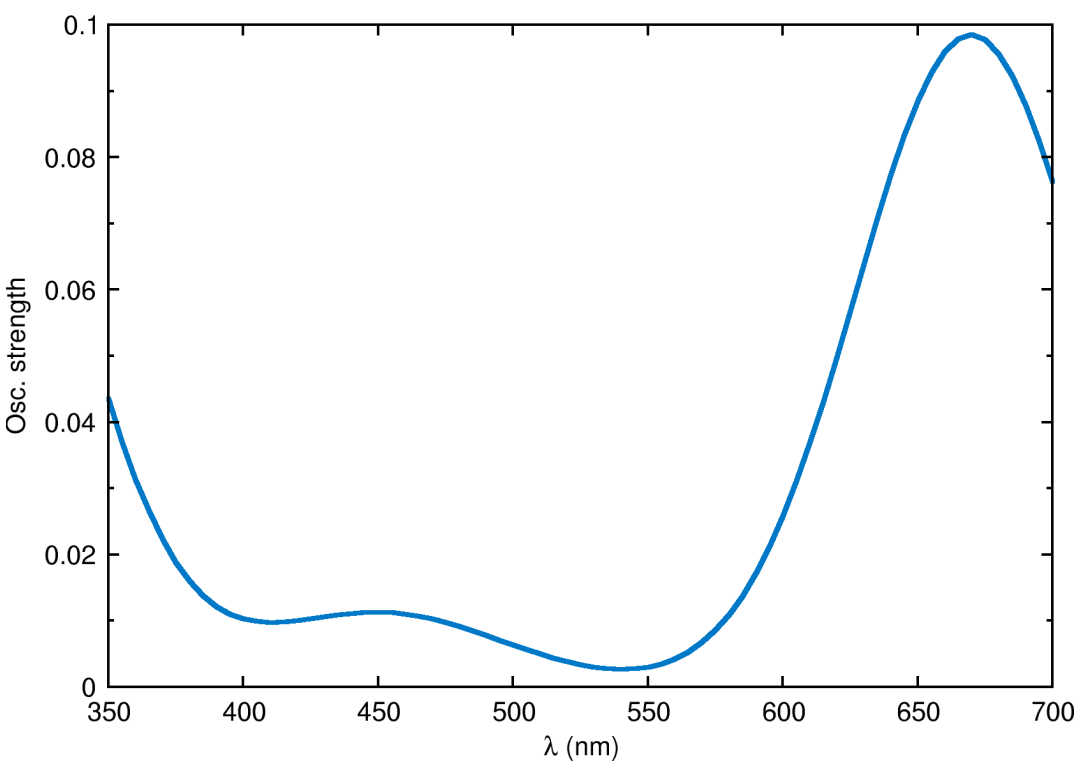

Figure S5 Transient Absorption Spectrum for $\mathrm{S}_{1} \mathrm{C}$ minimum

$$
\mathrm{T}_{1} \mathrm{~A}
$$

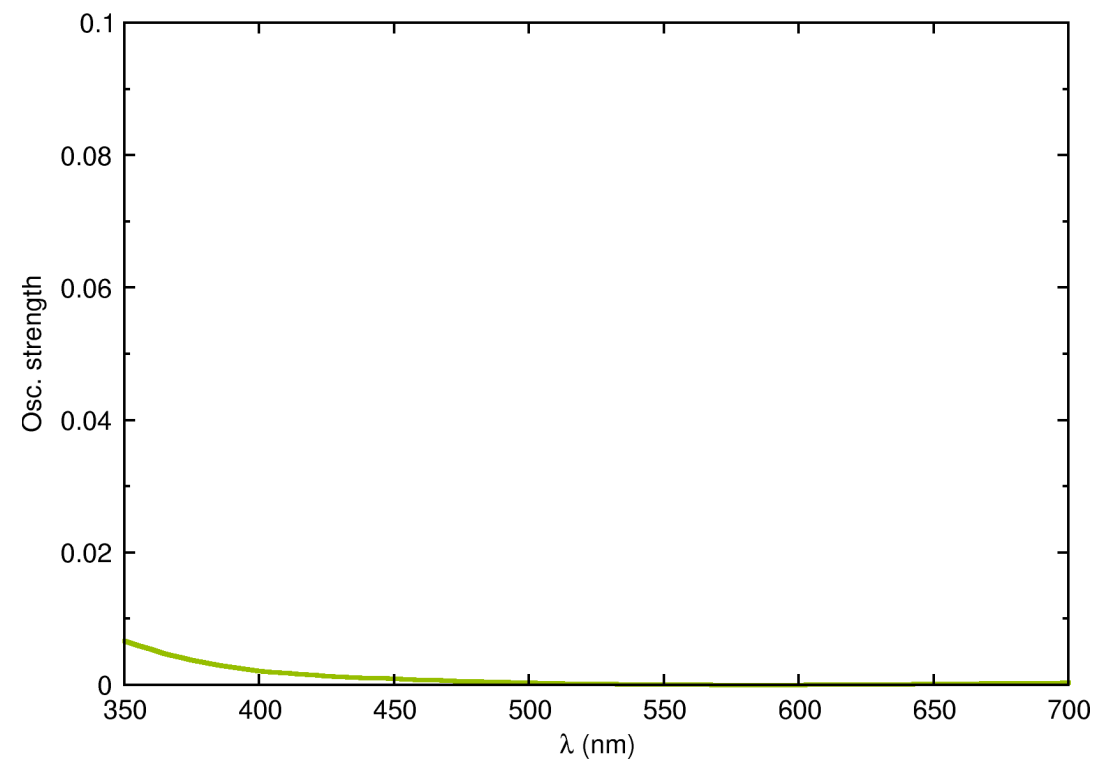

Figure S6 Transient Absorption Spectrum for $T_{1} A$ minimum 
$\mathrm{T}_{1} \mathrm{~B}$

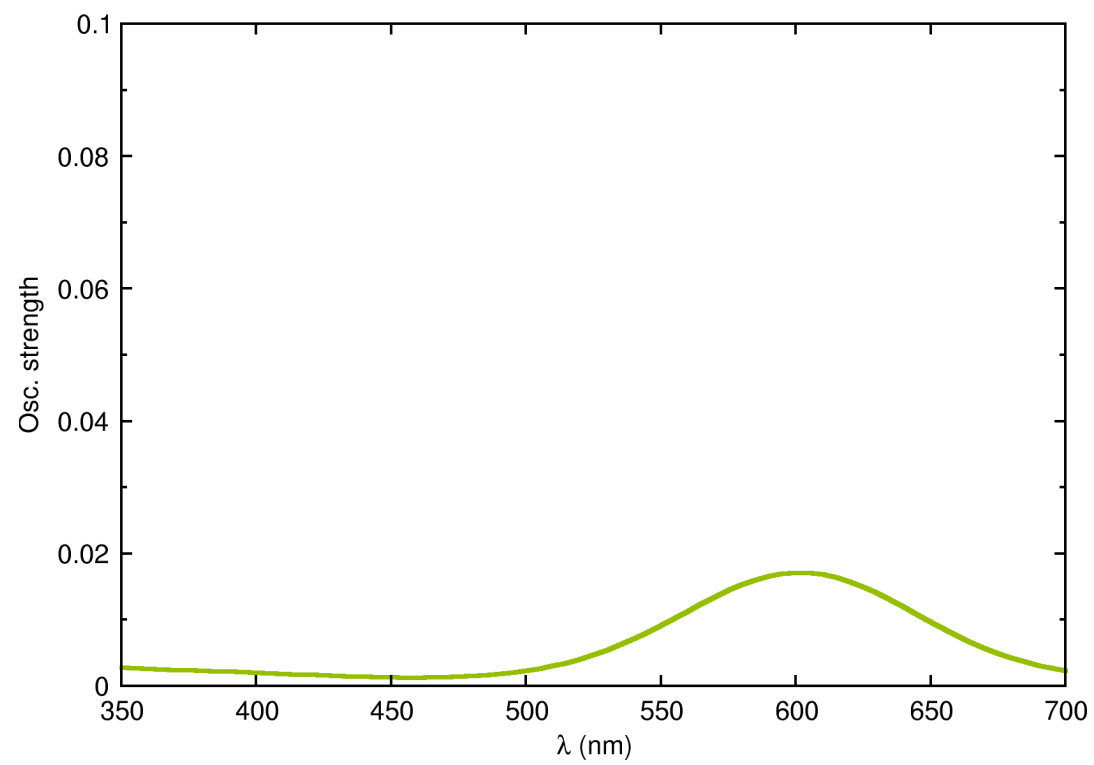

Figure S7 Transient Absorption Spectrum for $T_{1} B$ minimum

$$
\mathrm{T}_{1} \mathrm{C}
$$

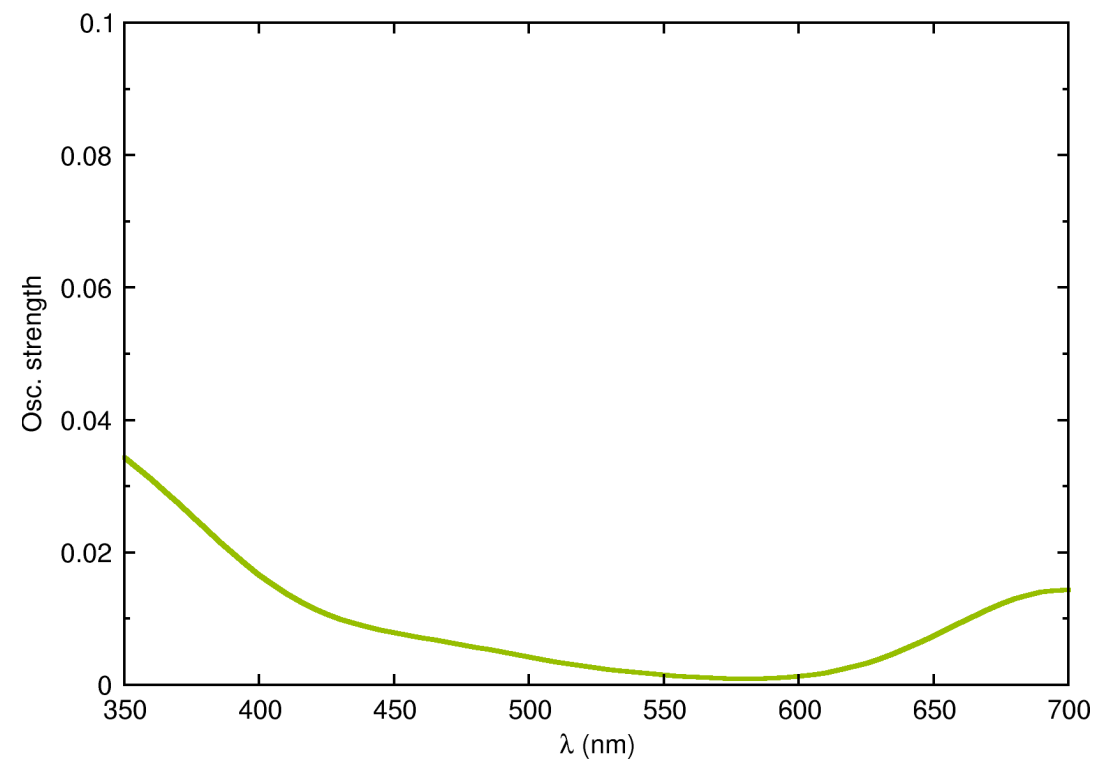

Figure S8 Transient Absorption Spectrum for $\mathrm{T}_{1} \mathrm{C}$ minimum 


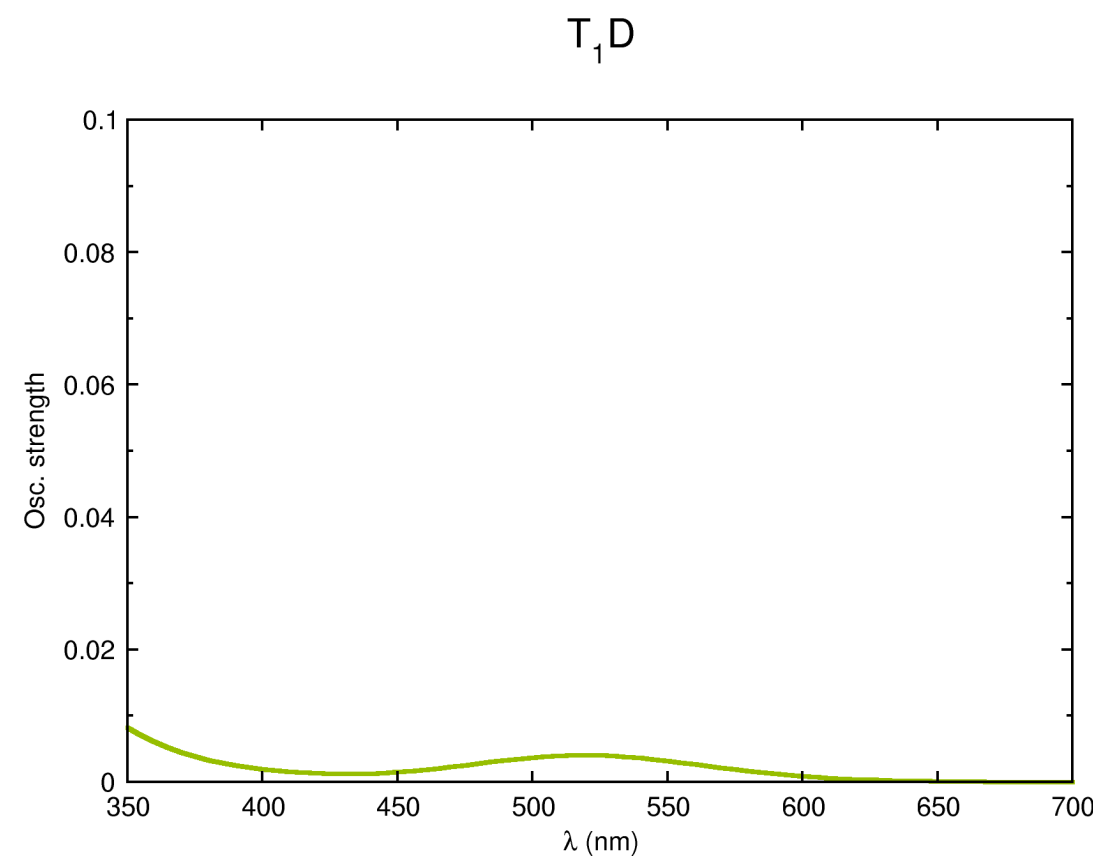

Figure S9 Transient Absorption Spectrum for $T_{1} D$ minimum

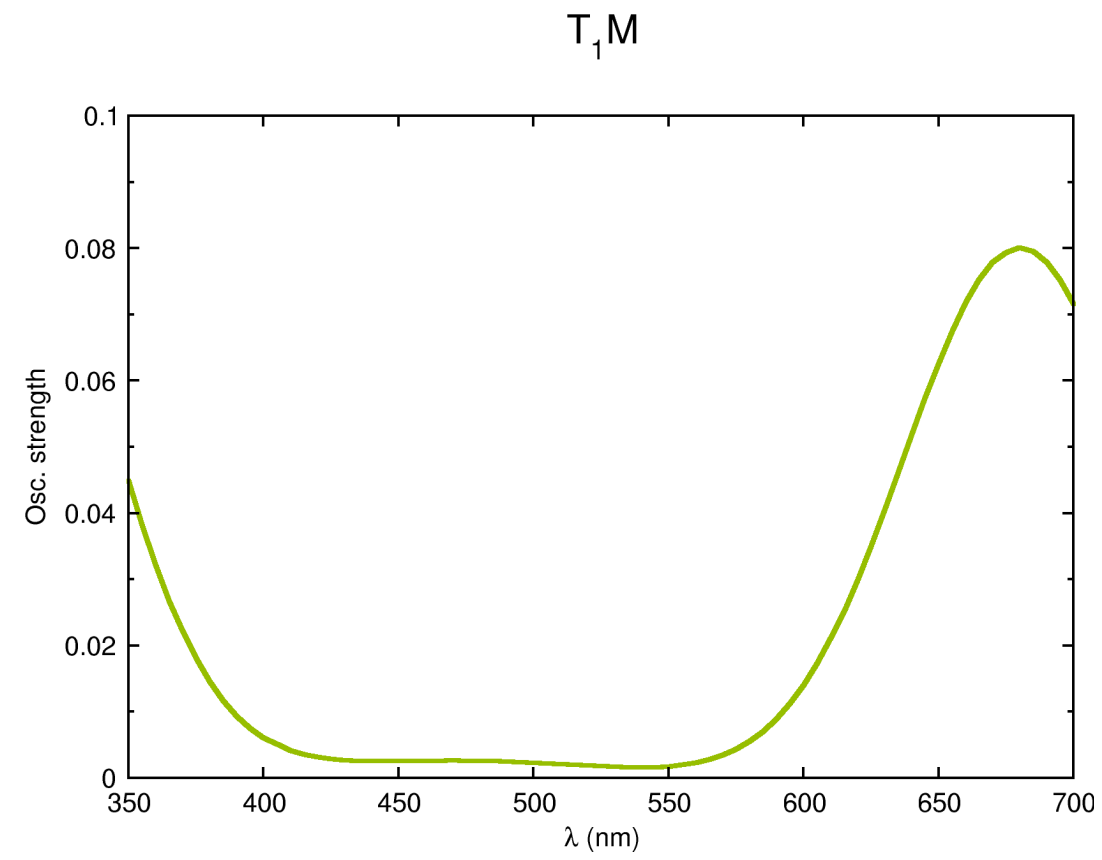

Figure S10 Transient Absorption Spectrum for $\mathrm{T}_{1} \mathrm{M}$ minimum 
$\mathrm{T}_{1} \mathrm{~N}$

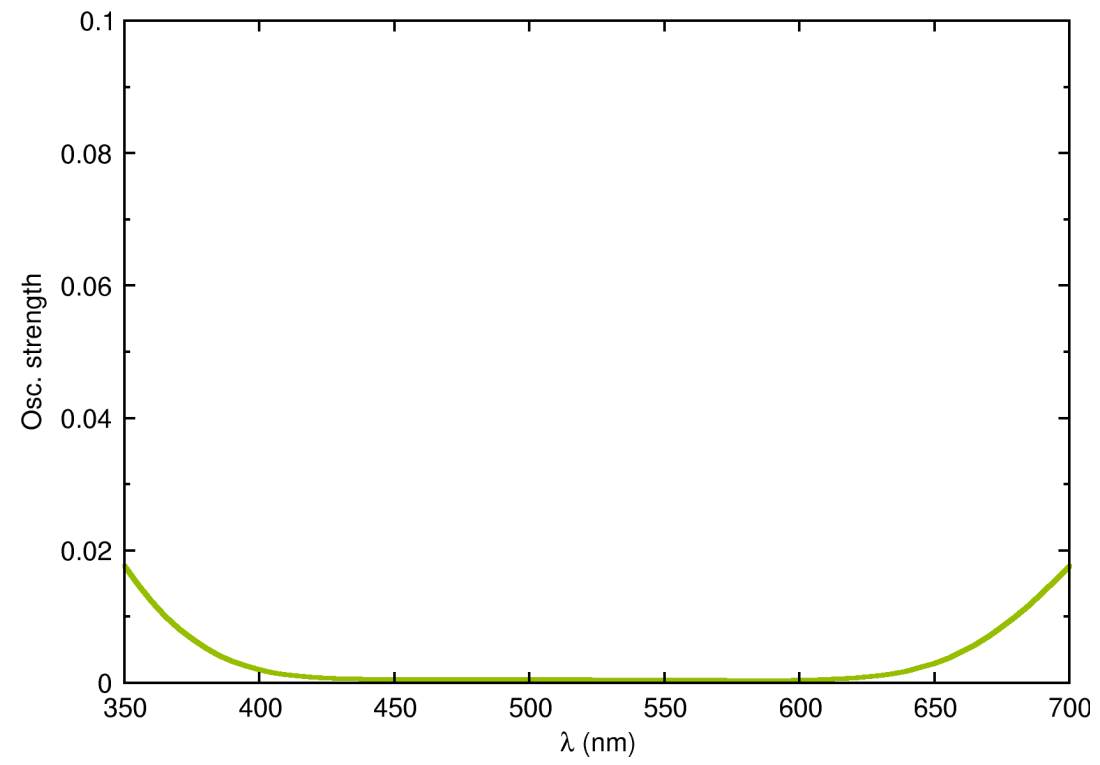

Figure S11 Transient Absorption Spectrum for $\mathrm{T}_{1} \mathrm{~N}$ minimum 


\section{Modeling of Evolution Associated Difference Spectra from Calculated Data}

For the modeling of the Evolution Associated Difference Spectra (EADS) the calculated spectrum for each of the excited state minima were linearly combined to produce an accurate replica. The search of the optimal coefficients was done automatically with a home-made program, which follows the procedure below described:

1) The program first transforms the calculated and experimental EADS spectra into new spectra with the same format (wavelength range and step). In order to produce smooth spectra, the intensity of new points are estimated by linear interpolation of two consecutive points (Figure S12 shows the comparison between the original EADS-1 spectrum and its formatted version).

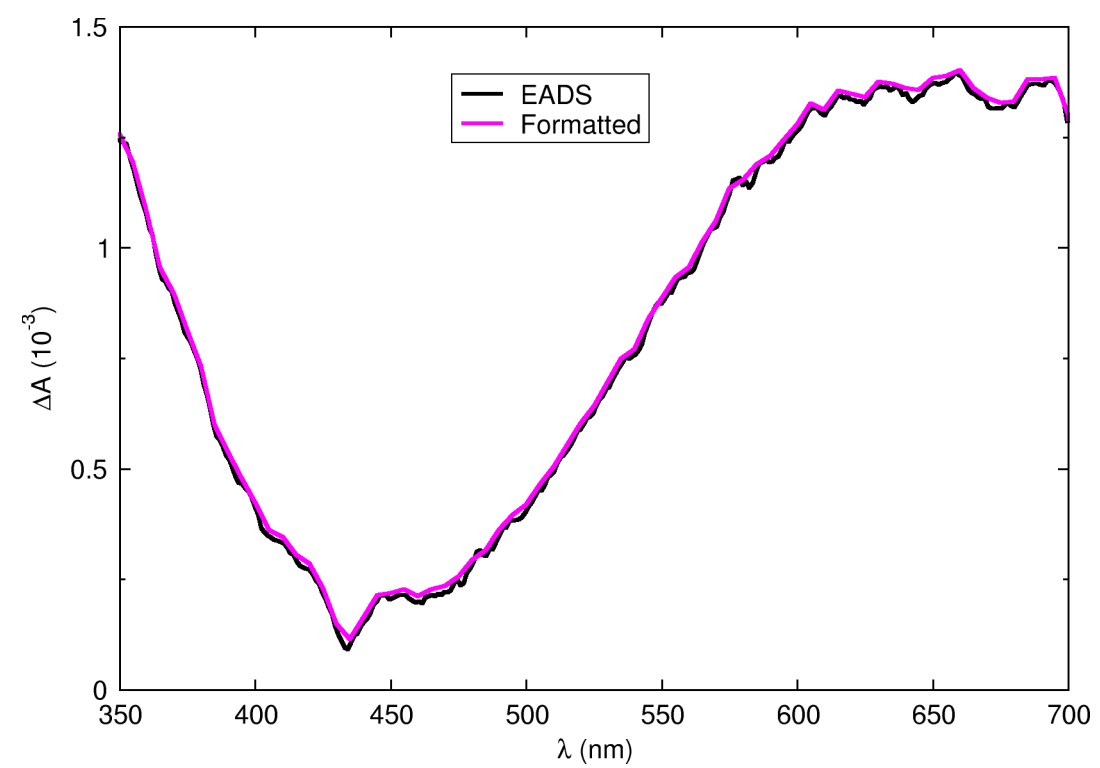

Figure S12 Comparison between the original EADS-1 (black) with its formatted version (purple)

2) Next, the program generates a guess for the EADS by linear combination of the calculated spectra using random coefficients. Then it normalizes the experimental EADS and the generated guess setting to 1 their maximum intensity.

3) The quality of the fitting procedure is given by the error factor $\delta$, calculated as:

$$
\delta=\sum_{n}\left|y_{\text {real }}-y_{\text {gen }}\right|
$$

where $\mathrm{n}$ is the wavelength, $\mathrm{y}_{\text {real }}$ is the intensity of the experimental EADS at that wavelength and $\mathrm{y}_{\text {gen }}$ is the intensity of the generated guess. Thus, the smaller the value of $\delta$, the better the quality of the fitting.

4) Steps 2 and 3 are repeated in a loop thousands of times, storing the best 20 fits (20 lowest values of $\delta$ ). The final result is obtained from the average of these 20 results.

The above procedure is preceded by a selection of the pool of potential minima to be used in the fitting of the EADS, based on the expected time evolution of the population according to the landscape of the potential energy surface. Thus, only singlets are used for the modelling of EADS-1, singlets and readily available triplets are employed for EADS-2 and only triplets are considered for EADS-3. In the following we report the results for the simulation of the experimental EADS from calculated data. 


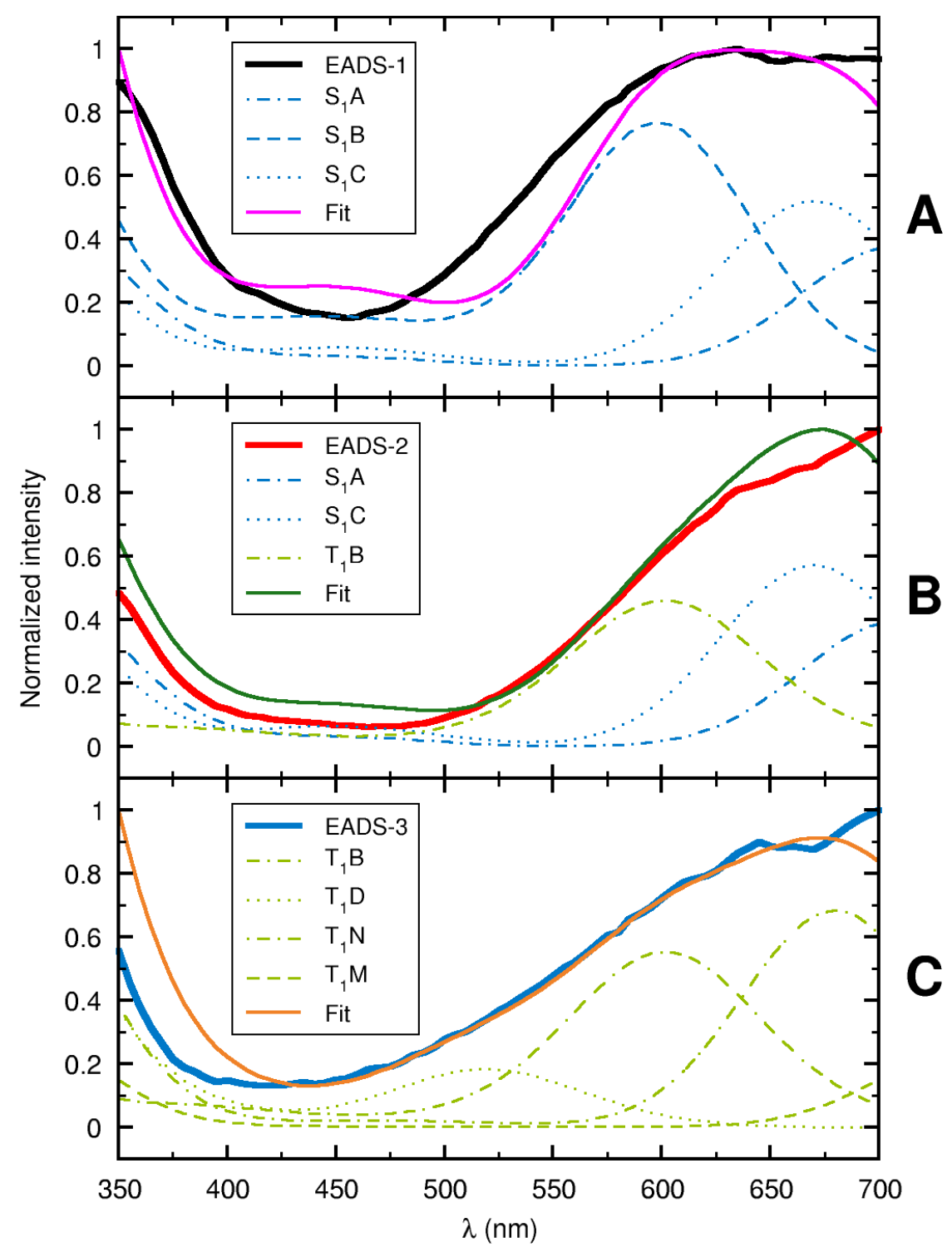

Figure S13 Superposition of the experimental and simulated evolution associated difference spectra. A (upper): EADS-1 (black line), fitted linear combination (purple line, $0.35 \mathrm{~S}_{1} A+0.49 \mathrm{~S}_{1} B+0.16 \mathrm{~S}_{1} C$ ). $B$ (medium): EADS-2 (red line), fitted linear combination (green line, $0.27 \mathrm{~S}_{1} A+0.13 \mathrm{~S}_{1} C+0.60 \mathrm{~T}_{1} B$ ). $C$ (lower): EADS-3 (blue line), fitted linear combination (orange line, $0.34 \mathrm{~T}_{1} B+0.48 \mathrm{~T}_{1} \mathrm{D}+0.09$ $\left.\mathrm{T}_{1} \mathrm{~N}+0.09 \mathrm{~T}_{1} \mathrm{~B}\right)$ 


\section{Experimental Methodology}

\subsection{Chemicals}

Pyrimidine ( $\geq 98 \%$ purity), acetonitrile (HPLC Grade, $\geq 99.93 \%$ purity) and cyclohexane (HPLC Grade, $>99.9 \%$ purity) were purchased from Sigma-Aldrich and used as received. Phosphate buffered saline (PBS) solutions at $\mathrm{pH}$ of 7.4 were prepared with a total concentration of $50 \mathrm{mM}$ from monosodium and disodium phosphate salts (99.0\% purity, Sigma-Aldrich). The $\mathrm{pH}$ was adjusted with $0.1 \mathrm{M}$ sodium hydroxide and phosphoric acid (Fisher Chemical, certified ACS grade $\geq 85 \% \mathrm{w} / \mathrm{w}$ ) to the desired $\mathrm{pH}$ ( $\pm 0.1 \mathrm{pH}$ units).

\subsection{Steady-State Spectroscopy}

The steady-state spectra of pyrimidine in PBS, pH 7.4, acetonitrile, and cyclohexane were taken using a UV-vis spectrometer (Cary 100 Bio Instrument, Agilent Technologies).

\subsection{Transient Absorption Spectroscopy}

The experimental setup and data analysis used for the broadband transient absorption pump-probe technique have been discussed in detail elsewhere. ${ }^{11-13}$ Briefly, generation of a $800 \mathrm{~nm}$ laser pulse at a $1 \mathrm{kHz}$ repetition rate with a pulse width of $100 \mathrm{fs}$ from a Libra-HE (Coherent, Inc.) was used. The fundamental 800 $\mathrm{nm}$ beam was split in two with $98 / 2$ beam splitter. The $2 \%$ portion was used to generate a white light continuum from 320 to $700 \mathrm{~nm}$ in a translating $2 \mathrm{~mm}$ calcium fluoride crystal. The $98 \%$ of fundamental beam was used to generate the excitation pulse. The $268 \mathrm{~nm}$ was generated using second-harmonic sum frequency generation using an optical parametric amplifier (TOPAS, Quantronix/light conversion). For excitation at $267 \mathrm{~nm}$, the second and third harmonic (SHG/THG) of the fundamental were generated using an extended FKE series SHG/THG kit (EKSMA optics). Briefly, excess high power $800 \mathrm{~nm}$ fundamental passes a zero order half wave plate, ensuring proper polarization. Next, SHG of the fundamental with orthogonal polarization, is generated from a type 1 barium borate (BBO) crystal. The $400 \mathrm{~nm}$ along with excess fundamental then pass through a group velocity delay compensation calcite plate, retarding the phases relative to one another. Afterward, both pass through a subsequent zero order half and full dual-wave plate for $800 \mathrm{~nm}$ and $400 \mathrm{~nm}$ respectively, allowing for phase and polarization matching conditions to be met. The two frequencies are then mixed in a second type 1 BBO crystal, generating the third harmonic of the fundamental of $267 \mathrm{~nm}$ excitation, where the individual wavelengths are separated through the use of dichroic mirrors at 45 degree angle of incidence.

Data processing made use of a home-made LabView program, while the target and global analyses were performed using the Glotaran software. ${ }^{14}$ The decay traces obtained from the femtosecond transient absorption data were globally fitted using a three-component sequential kinetic model, convoluted with a Gaussian instrument response function of $270 \pm 50 \mathrm{fs}$ (FWHM). The third kinetic component required a large lifetime (i.e., tens of ns) to satisfactorily fit the long-lived transient signals. It was presumed that the long-lived signal modeled by the third kinetic component decays exponentially. The evolution associated difference spectra (EADS) were extracted from the target and global analysis. ${ }^{15}$

Homogeneity of the solutions in a $2 \mathrm{~mm}$ fused silica cuvette were maintained using a magnetic stir bar throughout the experiments. Freshly prepared solutions were used if the lowest-energy absorption maximum decreases by more than $5 \%$, as judged by steady-state absorption spectroscopy, to ensure that the transient absorption signals were not contaminated by signals from any putative formation of photoproducts. 
In our laser experiments reported in the main text, an absorbance of 1.33 at the excitation wavelength was used to maximize the signal-to-noise ratio $(\mathrm{S} / \mathrm{N})$. This absorbance translates to a pyrimidine concentration of $0.014 \mathrm{M}$ in acetonitrile, which could raise a concern for the possibility of formation of aggregates of pyrimidine in the acetonitrile solution. Hence, we have performed additional transient absorption experiments exciting at $267 \mathrm{~nm}$ with a third harmonic generation setup (described in the Experimental Methods) that allow us to collect transient absorption data with a sevenfold lower concentration. We began by investigating if the concentration affects the absorption spectra of pyrimidine.
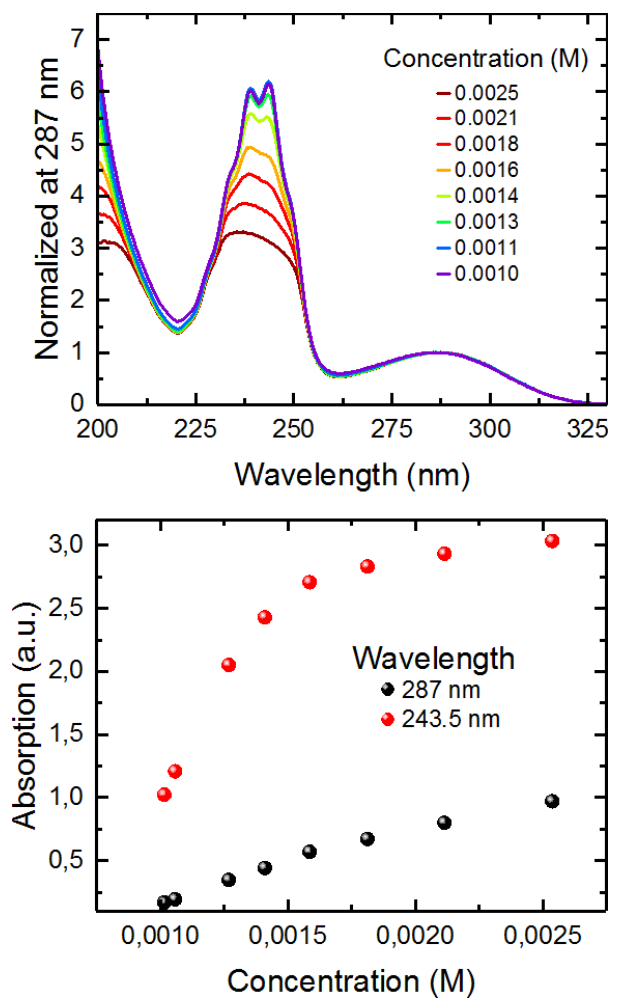

Figure S14 Top: Normalized absorption spectra of pyrimidine in the concentration range from 0.0010 to $0.0025 \mathrm{M}$ in acetonitrile. Bottom: Change in absorbance at 243.5 and $287 \mathrm{~nm}$ as a function of pyrimidine concentration. Note that the noticeable saturation of the absorption signal at $243.5 \mathrm{~nm}$ is primarily due to the saturation of the detector at absorbance higher than ca. 2 .

As can be observed in the top panel of Figure S14, no redshift or spectral broadening is observed for the lowest-energy absorption band of pyrimidine in the concentration range from 0.001 to $0.0025 \mathrm{M}$. At 287 $\mathrm{nm}$, a small decrease in linearity is observed when the concentration of pyrimidine is increased above ca. $0.0020 \mathrm{M}$ (see bottom panel of Fig. S14). This suggest that formation of aggregates at the concentration used to collect that data shown in Figures 2 and 3 may be a concern. Hence, we repeated the transient absorption experiments using a sevenfold lower concentration of pyrimidine $(0.0020 \mathrm{M})$ in order to scrutinize the possibility that aggregates may be affecting the excited state dynamics of the pyrimidine monomer. The absorbance of pyrimidine at the excitation wavelength of $267 \mathrm{~nm}$ is 0.21 , which is sevenfold lower than that used in Figures 2 and 3, requiring much longer collection time to achieve adequate $\mathrm{S} / \mathrm{N}$ (the solution was refreshed to avoid the putative contamination of the signals by the formation of photoproducts). As can be observed in Figures S15 and S16 below, the results presented in Figures 2 and 3 of the main text agree within experimental uncertainties to those collected at this lower concentration. We remark that at the $0.0020 \mathrm{M}$ concentration, any putative formation of aggregates is minimized (see Figure S14). 


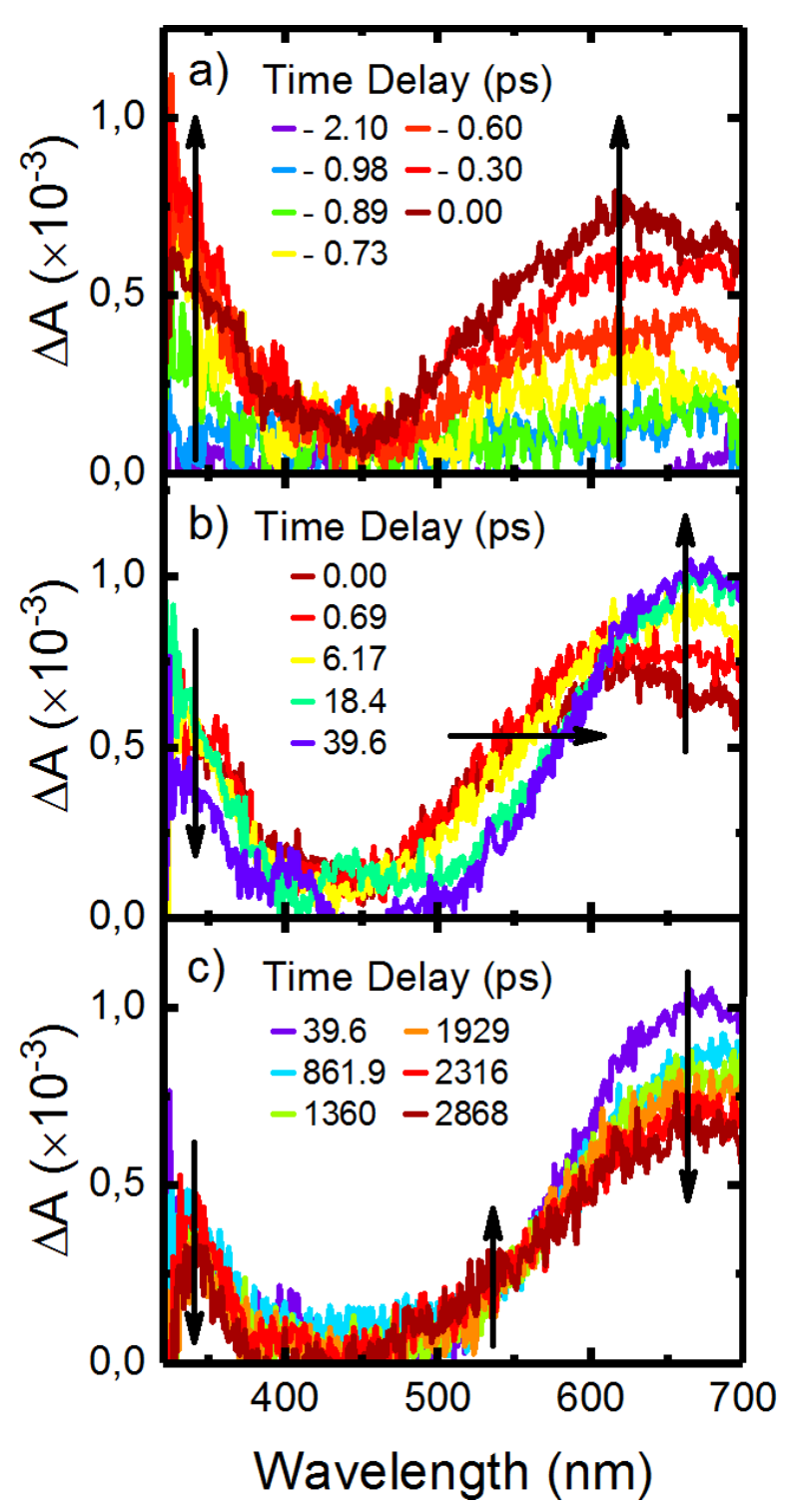

Figure S15 Transient absorption spectra for pyrimidine $(0.0020 \mathrm{M})$ in acetonitrile excited at $267 \mathrm{~nm}$.

The target and global analysis of the transient absorption data for a pyrimidine concentration of $0.0020 \mathrm{M}$ yielded lifetimes of $\tau_{1}=8.0 \pm 0.2 \mathrm{ps}$ and $\tau_{2}=790 \pm 40 \mathrm{ps}$, which are in reasonable agreement with the lifetimes obtained for a pyrimidine concentration of $0.0140 \mathrm{M}$ of $\tau_{1}=7.5 \pm 0.2 \mathrm{ps}$ and $\tau_{2}=2.1 \pm 0.1 \mathrm{~ns}$. We remark that the second lifetime has a significantly larger error than the statistical error the data analysis suggest because the transient data at both concentrations can be equally well fitted using a value of 790 ps or $2.1 \mathrm{~ns}$ for the second lifetime. This result is not surprising due to the limited temporal window used in this study of $c a .3$ ns. Hence, we report the averaged (and more conservative) lifetimes in the main text instead of either of the individual set of lifetimes obtained at each concentration. Therefore, the results at two significantly different concentrations suggest that any aggregates of pyrimidine formed at the $0.014 \mathrm{M}$ concentration is not appreciably affecting the excited state dynamics of the pyrimidine monomer in acetonitrile solution, or no significant amount of aggregates are formed at such a higher concentration in the first place. Further experiments in which the concentration of pyrimidine is varied systematically may be needed, but are outside the scope of the present investigation. 

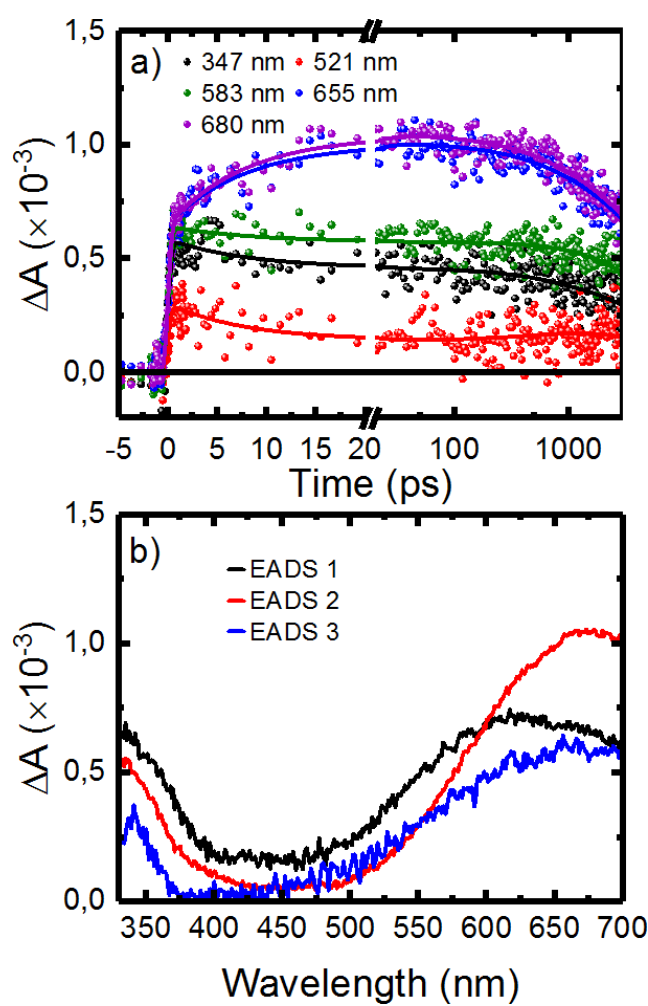

Figure $\mathbf{S 1 6}$ a) Selected kinetic traces for pyrimidine $(0.0020 \mathrm{M})$ in acetonitrile excited at $267 \mathrm{~nm}$. The fits represented are using a three component sequential kinetic model, where the third component required a large lifetime to satisfactorily fit the long-lived transient signal (i.e., tens of ns) using the Glotaran software. b) Evolution associated difference spectra (EADS) extracted using the Glotaran software. Note that these EADS are practically identical to those shown in Figure 3, which were obtained using a significantly higher pyrimidine concentration of $0.0140 \mathrm{M}$. 


\section{References}

1. Gaussian 09, Revision E.01, M. J. Frisch et al., Gaussian, Inc., Wallingford, CT, 2009.

2. (a) Andersson, K.; Malmqvist, P.-Å.; Roos, B. O., J. Chem. Phys. 1992, 96, 1218-1226. (b) Finley, J.; Malmqvist, P.-Å.; Roos, B. O.; Serrano-Andrés, L., Chem. Phys. Lett. 1998, 288, 299-306.

3. Roos, B. O., Ab Initio Methods in Quantum Chemistry; Wiley: Chichester, 1987; Vol. II.

$4 \quad$ Widmark, P.-O.; Malmqvist, P.-Å.; Roos, B. O., Theor. Chim. Acta 1990, 77, 291-306.

5. Shiozaki, T.; Györffy, W.; Celani, P.; Werner, H.-J., J. Chem. Phys. 2011, 135, 081106.

6. (a) Park, J. W.; Shiozaki, T.; J. Chem. Theory Comput. 2017, 13, 2561-2570. (b) Shiozaki, T., WIREs Comput. Mol. Sci. 2017, 8, e1331.

7. Werner, H.-J. et al., WIREs Comput. Mol. Sci. 2012, 2, 242-253.

8. Aquilante, F. et al., J. Comput. Chem. 2010, 31, 224-247.

9. Yanai, T.; Tew, D.; Handy, N., Chem. Phys. Lett. 2004, 393, 51-57.

10. Barone, V.; Cossi, M.; Tomasi, J., J. Chem. Phys. 1997, 107, 3210-322.

11. Reichardt, C.; Vogt, R. A.; Crespo-Hernández, C. E., J. Chem. Phys. 2009, 131, 224518.

12. Pollum, M.; Jockusch, S.; Crespo-Hernández, C. E., J. Am. Chem. Soc. 2014, 136, 17930-17933.

13. Brister, M. M., Crespo-Hernández, C. E., J. Phys. Chem. Lett. 2015, 6, 4404-4409.

14. Snellenburg, J.J.; Laptenok, S. P.; Seger, R,; Mullen, K. M.; van Stokkum, I. H. M., J. Stat. Software 2012, 49, 1-22.

15. van Stokkum, I. H. M.; Larsen, D. S.; van Grondelle, R., Biochim. Biophys. Acta 2004, 1657, 82-104. 